\title{
Galactic Haloes from Self-Interacting Neutrinos
}

\author{
Richard B. Holmes \\ The Boeing Company, Chicago, USA \\ Email: rholmes001@aol.com
}

How to cite this paper: Holmes, R.B. (2020) Galactic Haloes from Self-Interacting Neutrinos. Journal of Modern Physics, 11, 854-885. https://doi.org/10.4236/jmp.2020.116053

Received: May 10, 2020

Accepted: June 1, 2020

Published: June 4, 2020

Copyright $\odot 2020$ by author(s) and Scientific Research Publishing Inc. This work is licensed under the Creative Commons Attribution International License (CC BY 4.0).

http://creativecommons.org/licenses/by/4.0/

\begin{abstract}
The objective of this research is to provide an explanation of galactic haloes using established particles and forces using recent theoretical developments. Light fermions, with masses on the order of $1 \mathrm{eV} / \mathrm{c}^{2}$, are not a leading candidate for dark matter because of their large free-streaming scale length and their violation of the Tremaine-Gunn bound. With a self-interaction of fermions, the free-streaming scaling length is reduced, and the tenets of the Tremaine-Gunn bound are not applicable. Binding of neutrinos via a feeble $\mathrm{SU}(3)$ force is considered as a model for such interactions. The assumed sum of masses of the three neutrino flavors is $0.07 \mathrm{eV} / \mathrm{c}^{2}$. The resulting form of matter for such bound neutrinos is found to be a degenerate Fermi fluid. Pressure-equilibrium approaches applied to this fluid provide cuspy solutions and match observationally-inferred profiles for galactic haloes. Such approaches also match the observed total enclosed mass for galaxies similar to the Milky Way. The computed structures are found to be stable. The hypothesis is considered in view of observationally-inferred halo-halo interactions and gives results that are consistent with the observed Bullet cluster halo interaction. The theory gives agreement with observationally-inferred properties of dark matter near earth. Questions related to interaction rates, consistency with SN1987a data, the cosmic microwave background, the issue of $\mathrm{SU}(3)$ interactions between neutrinos and quarks, free-streaming after neutrino decoupling, and dark-matter abundance are addressed in a companion paper.
\end{abstract}

\section{Keywords}

Galactic Haloes, Dark Matter, Halo-Halo Interactions

\section{Introduction}

Dark matter (DM) has been postulated to take many forms, including hot dark matter [1] [2], warm dark matter [3] [4], massive compact halo objects [5] [6] 
[7], weakly interacting massive particles [3], and cold dark matter (CDM) [8] [9]. The conventional picture of neutrinos as dark matter was ruled out early [10]. The best current model for CDM is the $\Lambda$-CDM model [11], which assumes a certain fraction of the matter in the universe is cold (non-relativistic), non-interacting, and stable [12]. However, the model has a number of open issues [13] [14]. In particular, there is no explanation for dark matter. Massive neutrinos as inserted in the Standard Model have been postulated for dark matter [1] but rejected because in the conventional view neutrinos are almost always relativistic particles so any structure would diffuse away quickly and could not lead to the structures observed in the universe today. There are currently many hypothetical explanations for dark matter. Extensions to the Standard Model have been proposed to explain dark matter, dark energy, and other aspects of cosmology, e.g. [15] [16] [17] [18] [19]. QCD-like and nuclear like forces have recently been suggested for self-interacting dark matter, e.g. [20] [21]. In this paper, an SU(3) force applied to neutrinos is hypothesized as an explanation for galactic haloes, and by extension, DM.

The standard view posits that DM was in thermal contact with visible matter in the early universe when the temperature was much greater than the DM mass. In those eras, the DM number density would be comparable to photon number density. If the DM number density was still comparable to the photon number density when it froze out, it would overproduce the observed amount of DM mass when the DM particle mass is more than about $1 \mathrm{eV} / \mathrm{c}^{2}$ [22]. Thus, there is a need to deplete the abundance of any massive dark matter that is sufficiently cold at recombination. This is the path that leads to DM particles that are required to be largely annihilated in the early universe. An interaction energy of $100 \mathrm{GeV} \sim k T$ leads to a weak interaction cross section that when multiplied by the density and velocity at that time leads to a decay rate comparable to the expansion rate. Here $k$ is Boltzmann's constant and $T$ is the temperature. This would result in freeze-out at that early time in the primordial universe for the corresponding particle mass. Hence DM masses of order $100 \mathrm{GeV}$ would be candidates for DM under these standard assumptions. These would become extremely cold (non-relativistic) over the eons as the universe expanded.

Thus, the most anticipated masses of cold dark matter are associated with weakly interacting massive particles (WIMPs), with masses in the range of 10 to $100 \mathrm{GeV}$. However, in the absence of significant evidence of massive DM, the community is looking to lighter alternatives. To avoid the free-streaming issue with lighter DM, one approach is for DM to bind and/or cool early in the history of the universe. One might look for particles and/or forces for which such cooling and binding occurs. Such behavior might be analogous to the binding via $\mathrm{SU}(3)$ of quarks into hadrons in the early universe.

In the past 4 decades, computationally-intensive approaches have investigated the consistency of lighter DM with astronomical observations. Such investigations began with [10] regarding the possibility of neutrinos for dark matter. Reference [23] investigated consistency of dark matter with Lyman- $\alpha$ lines. Early 
modeling on light DM and self-interacting DM (SIDM) include [24] with elastic collisions and [25] on gravothermal collapse. Reference [26] simulated subhaloes and [27] considered the impact of fermions. More recently, [28] [29] performed extensive modeling of galaxy formation within larger structures in the Universe, [30] investigated an effective theory for small scale structure, [20] [31] considered SIDM and halo interactions, [32] considered the impact of SIDM on structure and self-assembly history, and [33] modeled SIDM that includes inelastic scattering. There have been recent reviews of SIDM by [21] and of the larger topic of dark matter haloes and subhaloes by [34]. Most of the more recent papers consider particles with masses of the order of a $\mathrm{keV} / \mathrm{c}^{2}$, as is consistent with observationally inferred values from the latest Lyman- $\alpha$ forest absorption measurements [35] and gravitational lensing measurements [36]. However, both these observationally-inferred mass bounds and the other aforementioned papers rely on assumptions that are not consistent with relativistic fermions with a mass of order $0.1 \mathrm{eV}$ that bind into a number of species of heavier particles in the primordial era, which then further bind into macroscopic structures later.

Section 2 considers the basic application of the SU(3) hypothesis to neutrinos in the early universe. Section 3 compares the predictions of the hypothesis to the observed small- and large-scale structure of the universe. Section 4 discusses the hypothesis in the context of the Tremaine-Gunn bound. Section 5 computes the properties of the proposed bound neutrinos near earth. Section 6 discusses the self-interacting properties of the proposed DM in haloes and between haloes. Section 7 summarizes the overall findings of this effort.

\section{The Feeble SU(3) Hypothesis and Neutrinos}

The hypothesis of a feeble form of $\mathrm{SU}(3)$ for the neutrino family is not immediately obvious from the standard model. From the standard model one might expect an interaction energy of the order of the QCD energy scale, $\sim 200 \mathrm{MeV}$ [37]. However, motivation can be found for a feeble SU(3) interaction between neutrinos in a modest extension of the standard model [38]. In this extension, $\mathrm{SU}(3)$ is not precluded for the neutrino family. In this theory, neutrino oscillations are direct evidence that neutrinos form bound states via $\mathrm{SU}(3)$. As shown in Appendix A, this extension also provides a means for estimating the neutrino interaction strength by scaling the quark interaction strength by $\left(m_{v} / m_{q}\right)^{2}$, where $m_{v}$ is the mass of the highest-mass neutrino and $m_{q}$ is the mass of the highest-mass quark of the up or down families. This scaling applies when the interaction involves relativistic neutrinos. Using $m_{v}$ of about $0.055 \mathrm{eV} / \mathrm{c}^{2}$ for the tau neutrino for minimal neutrino masses and the normal hierarchy [39] one finds a reduction in the SU(3) strength for relativistic neutrinos by a factor of $1.73 \times 10^{-22}$ to $1.01 \times 10^{-25}$ using the bottom quark or top quark, respectively, for $m_{q}$. This theory also has the property that in addition to the 8 massless gluons, there are 15 massive Goldstone bosons (massive gluons) for each family with gluon energies of the order of $m_{v} c^{2}$. These massive gluons satisfy most of the cri- 
teria proposed by [17] for a correction to the observed effective number of neutrinos. This extended form of $\mathrm{SU}(3)$ will be denoted $\mathrm{SU}(3)_{v e}$ and the standard form of SU(3) applied to the neutrino family will be denoted SU(3) ${ }_{v s}$ It will be seen that both forms of $\mathrm{SU}(3)$ are consistent with galactic halo data, but the former provides estimates of binding energies and particle masses whereas the standard SU(3) does not.

What are the consequences of a feeble analogue of quark confinement with neutrinos? Neutrinos typically start out as ultra-relativistic isolated particles near infinite redshift. In the early universe, the neutrinos would have formed a neutrino/neutrino-gluon plasma, much as would have occurred with quarks and gluons. By analogy with quarks, neutrinos would be bound into "mesonic" or "baryonic" neutrinos, and they would then remain confined to the present day. When excited with sufficient energy, perhaps by hot stellar neutrinos, such bound states might "hadronize" to form additional bound neutrinos while remaining confined, analogous to the behavior of quarks we see today.

Table 1 shows that the partition of energy using the standard approach, e.g. [40], applied to the hypothesis. It is seen that both forms of SU(3) can supply a fraction of the mass-energy that corresponds to the modern estimate of the fraction of mass-energy in the dark matter sector, about 84\% [41]. The confined baryonic neutrinos would hold the vast reservoir of the mass-energy of SU(3) neutrino gluons shown in Table 1.

When would the above interactions terminate? Such neutrinos should "hadronize" when their total center-of-mass (CM) collision energy exceeds about $4 m_{\nu \mu} c^{2}$, where $m_{v \mu}$ is the mass of the muon neutrino, by analogy with the quark families, assuming the normal hierarchy. Hence this limits the maximum kinetic energy to about $m_{\nu \mu} c^{2}$ for each particle. Also, there is no interaction pathway to hadronize when the total CM energy of any neutrino state is less than $4 m_{v e} c^{2}$. Here $m_{v e}$ is the mass of the lowest-mass neutrino, the electron neutrino in the normal hierarchy. This gives a range of kinetic energies from $m_{v e} c^{2}$ to $m_{v \mu} c^{2}$ in the CM frame, and about a factor of 2 more in the local rest frame in the early universe. From our current knowledge of neutrino masses in the normal hierarchy,

Table 1. Energy density degrees of freedom in the early universe with $k T$ at $\sim 1 \mathrm{MeV}$ assuming standard $\mathrm{SU}(3)$ for neutrinos, $\mathrm{SU}(3)_{n s}$, and an extended version, $\mathrm{SU}(3)_{n e}$.

\begin{tabular}{ccc}
\hline Particle & $\begin{array}{c}\text { Degrees of Freedom, } \\
\text { SU }(3)_{v s}\end{array}$ & $\begin{array}{c}\text { Degrees of Freedom, } \\
\text { SU }(3)_{v e}\end{array}$ \\
Electron family & $(1)(4)=4(\times 7 / 8)$ & $(1)(4)=4(\times 7 / 8)$ \\
Photons & 2 & 2 \\
Neutrino family & $(3)(2)(3)=18(\times 7 / 8)$ & $(3)(2)(3)=18(\times 7 / 8)$ \\
Neutrino family gluons & $(8)(2)=16$ & $(15)(3)+(8)(2)=61$ \\
Total degrees of freedom & 37.25 & 82.25 \\
Total degrees of freedom in neutrino sector & 31.75 & 76.75 \\
\% Degrees of freedom in neutrino sector & $83.9 \%$ & $93.3 \%$ \\
\hline
\end{tabular}


$m_{v e} c^{2} \sim 0.005 \mathrm{eV}$ and $m_{v \mu} c^{2} \sim 0.01 \mathrm{eV}$ [39]. The implied approximate range of steady-state mean thermal kinetic energy is $(3 / 2) k T=0.01$ to $0.02 \mathrm{eV}$, i.e. $k T=$ 0.007 to $0.014 \mathrm{eV}$. For the inverted hierarchy, the range of $k T$ is 0.007 to $0.07 \mathrm{eV}$ assuming appropriate adjustments of the above masses. These estimates for kinetic energies would apply at the end of the hypothesized period of neutrino binding into baryonic neutrinos; further evolution would be expected as the universe expands.

\section{Consistency of the Hypothesis with the Size and Shape of Galactic Haloes}

\subsection{Equations and Inputs for Spatial Structure of DM}

The spatial density profile derived from the hypothesis of bound neutrinos is compared to observationally-inferred galactic halo structures. The characteristic scales sizes of galaxies for ordinary radiant matter (OM) are 1 - $200 \mathrm{kpc}$ depending on the galaxy and somewhat larger for the associated haloes [42].

To analyze the spatial distribution of low-energy neutrinos or other weakly interacting particles, an N-body simulation [20] [31] or the Vlasov equation is typically preferred [43]. Herein, two simplified governing equations are considered for DM density profiles. The first is the standard equation for hydrostatic equilibrium in a spherically-symmetric geometry. This equation is known to be inadequate for dark matter haloes, as further shown below. This equation is given by

$$
(1 / \rho)(\mathrm{d} P / \mathrm{d} r)=-m_{b v} M_{e n c}(r) G / r^{2} .
$$

Here $\rho$ is the number density of DM, $P$ is the pressure, $r$ is the radius, $m_{b v}$ is the mass of a DM particle, $M_{\text {enc }}(r)$ is the enclosed mass, and $G$ is the gravitational constant. This equation can be solved using the well-known Lane-Emden formulation [44] if pressure is a function of density only:

$$
P=c_{\gamma} \rho^{\gamma},
$$

where $c_{\gamma}$ is a constant for a given polytropic exponent $\gamma$. An inhomogeneous form of the Lane-Emden equation can be used when OM is present. As shown later in this section, initial calculations using Equation (1) with a galaxy similar to the Milky Way for OM do not match key published results from simulations or inferences from observations for DM. In particular, the solutions have no cusp at the origin, and are a poor match to the standard de-projected Sersic or Einasto profiles based on observations [42] [45]-[51]. This is found to be true for any polytropic exponent between $4 / 3$ and 2 .

To address this, a generalization of Equation (1) is used. The derivation and the properties of the resulting equation are given in [52]. The basic result is

$$
\begin{aligned}
& (1 / \rho) \mathrm{d} P / \mathrm{d} r+(1 / \rho)(\mathrm{d} \rho / \mathrm{d} r)\left[m_{b v} M_{e n c}(r) G / r\right] \\
& =-m_{b v} M_{e n c}(r) G / r^{2}-(\mathrm{d} P / \mathrm{d} \rho) / r
\end{aligned}
$$

when pressure is assumed to be a function of density only. It can be seen that 
this equation reduces to Equation (1) when the second terms of both sides of the equation are negligible. When all terms are included, Equation (3) is seen to give a $1 / r$ dependence for the density. Equation (3) with a total mass constraint and a density constraint is the most justifiable based on theoretical considerations, comparisons with data, and comparisons with others' calculations and simulations. This best-justified result is given by

$$
\rho(r)=\left\{\begin{array}{l}
\rho_{0} \text { in a spherical region about the origin of radius } r_{0} \text {, or } \\
\rho(r) \text { satisfies } \frac{\mathrm{d} \ln \rho}{\mathrm{d} \ln r}=-1-2\left(\frac{r}{r_{c}}\right)\left(\frac{\rho_{c}}{\rho}\right)
\end{array}\right.
$$

In this equation, $r_{c}$ is the cutoff radius and $\rho_{c}$ is the cutoff number density where the density drops due to the mass constraint. The last key equation used for calculations of haloes is the Fermi-Dirac equation for the number density given temperature $T$ and particle mass $m_{b v}$ :

$$
\rho=n_{s} /\left(2 \pi^{2} \hbar^{3}\right) \int p^{2} \mathrm{~d} p /\left[\exp \left(\left\{\left[(p c)^{2}+\left(m_{v} c^{2}\right)^{2}\right]^{1 / 2}-m_{v} c^{2}-\mu_{v}\right\} / k T\right)+1\right],
$$

where $n_{s}$ is the number of spin states and $p$ is the fermion momentum. Note that the general form is used, applicable to both relativistic and non-relativistic states. The chemical potential is denoted by $\mu_{\nu}$ and will be estimated later. Note that for trapped neutrinos the chemical potential may be non-zero, as with ordinary bound matter. Equation (5) yields the result $P=\left(1.914 \hbar^{2} / m_{b v}\right) \rho^{5 / 3}$ for non-relativistic fermions with two spin states. This equation is used to set the density at the origin, and then Equation (1) and Equation (2) or Equation (4) are used to generate a spatial profile. The resulting solution of Equation (4) for number density $\rho(r)$ can be expressed as a function of its inputs, $\rho(r)=\rho\left(r, \rho_{0}, r_{0}, \rho_{c}, r_{c}\right)$. Using Equation (5), $\rho(r)$ can also be expressed as a function of temperatures and mass, $\rho(r)=\rho\left(r, T_{0}, r_{0}, T_{c}, r_{c}, m_{b v}, \mu_{v}\right)$, where $T_{0}$ is the temperature at the origin, $T_{\mathrm{c}}$ is the temperature corresponding to the cutoff density $\rho_{c}$, and the other variables are defined above. This notation will be used below.

To solve Equation (1) and Equation (2), an equation of state that relates pressure to density and temperature must be chosen. In many treatments in astronomy and astrophysics, the pressure is a function of density only as in Equation (2), yielding an implicit relationship between temperature and density. One natural choice that relates temperature and density is Equation (5). The relativistic and non-relativistic versions have been used to derive the equation for the density in dwarf stars [53] [54]. The relativistic and non-relativistic versions give polytropic exponents of $4 / 3$ and $5 / 3$, respectively. The latter choice might be expected to be a good one for a cool fermionic gas.

As another option for the polytropic exponent, one may look for physical models in the literature for chargeless baryonic particles in a gravitational field. Such a model can be found in treatments of neutron stars. Treatments of neutron stars typically use a polytropic exponent ranging from $3 / 2$ to 2 , with near 2 as the most common and most likely choice [55] [56] [57]. Such an exponent is 
also present in the van der Waals equation of state. Thus, a choice $\gamma=2-\varepsilon$ with $0<\varepsilon \ll 1$ is also considered below for galactic haloes.

To complete the initial conditions, the density at $r=0$ must be known or assumed. Given the discussions at the end of Section 2, one expects $k T$ to be in the range of 0.007 to $0.07 \mathrm{eV}$ near galactic centers at the time of halo formation. This range of temperatures corresponds to a range of densities given by Equation (5).

Such a range of mean kinetic energies is considered in combination with the possible range of masses of baryonic neutrinos. As given in Appendix A, the range of masses considered for baryonic neutrinos is 0.025 to $0.6 \mathrm{eV} / \mathrm{c}^{2}$. A possible range of key properties of a fermion gas is shown in Table 2. In particular, the table assumes a temperature of $0.0134 \mathrm{eV} / \mathrm{k}=155 \mathrm{~K}$, which is found to give good agreement with density profiles of galactic haloes inferred from data, as will be shown in the next section. From Equation (5) one then obtains a number density, shown in the second column (with the chemical potential initially set to zero). The third column shows the root-mean-square (RMS) velocity, $v_{r m s}$, which is obtained from the well-known relation between relativistic velocity and kinetic energy. The fourth column shows the energy density computed using $\rho m_{b v} c^{2} /\left[1-\left(v_{r m s} / c\right)^{2}\right]^{1 / 2}$.

From this table, one sees a range of mildly relativistic velocities. These velocities are not consistent with gaseous dark matter that is bound in galactic haloes solely by gravitational attraction, since the escape velocity for a galaxy is typically of the order of $500 \mathrm{~km} \cdot \mathrm{sec}^{-1}$ [41]. To address this issue, one may recall the analogous states of baryonic quarks, which form atomic nuclei or neutron stars, as mentioned above. In the absence of electrostatic repulsion, nuclei can be of unbounded size, according to the Weizsacker model [58]. Hence, one might surmise that baryonic neutrinos form a similar macroscopic state in which very weak binding occurs (but not binding with other forms of matter).

Such a binding energy between neutrinos in baryonic states would need to be at least the mean kinetic energy, i.e. the value of about $0.02 \mathrm{eV}$ in order to avoid the free-streaming issue. To estimate such a binding energy, one might consider the nuclear binding energy of about $15 \mathrm{MeV}$ per nucleon and use the ratio of the

Table 2. Tentative range of key properties of a baryonic neutrino medium near a galactic center ${ }^{\mathrm{a}}$.

\begin{tabular}{cccc}
\hline Mass $\left(\mathrm{eV} / \mathrm{c}^{2}\right)$ & Number Density, $\rho_{0, m V}\left(\times 10^{15} \mathrm{~m}^{-3}\right)$ & $\mathrm{RMS}$ velocity/c & Energy Density $\left(\mathrm{GeV} \cdot \mathrm{cm}^{-3}\right)$ \\
\hline 0.025 & 0.185 & 0.83 & 0.0083 \\
0.05 & 0.36 & 0.70 & 0.0250 \\
0.10 & 0.81 & 0.55 & 0.0966 \\
0.2 & 2.01 & 0.42 & 0.441 \\
0.3 & 3.55 & 0.35 & 1.13 \\
0.4 & 5.34 & 0.30 & 2.23 \\
0.6 & 9.58 & 0.25 & 5.91 \\
\hline
\end{tabular}

${ }^{a}$ Assumes a mean kinetic energy of $0.02 \mathrm{eV}$, corresponding to a temperature of $155 \mathrm{~K}$ as described in text. 
mass of a baryonic neutrino to that of a neutron. Such a scaling gives a binding energy of about $(15 \mathrm{MeV}) \times(0.4 \mathrm{eV}) /(939 \mathrm{MeV})=0.0064 \mathrm{eV}$ between neutrino-based baryons. This linear scaling of baryonic binding with particle mass is partially justified by Appendix A. One might also envision a binding between up to six neighboring baryonic neutrinos (divided by two since bonds are shared), leading to a total binding energy up to about $0.0192 \mathrm{eV}$, which is comparable to the mean kinetic energy of $0.02 \mathrm{eV}$ given above. A more detailed ab initio calculation is beyond the scope of this paper. However, these estimates of binding energy and thermal kinetic energy are roughly consistent with what is required from the virial theorem for a medium in equilibrium $(\langle K . E\rangle=.n\langle V\rangle$ with $n=-1$ for massless gluons or gravity and $n \sim 1$ for a $b r$ inter-particle potential, where " $\langle$ K.E. $\rangle$ " denotes a time average of the total kinetic energy and " $\langle V\rangle$ " denotes the time average of the total potential energy).

With estimates for a binding energy and a range of densities, one may then estimate a chemical potential to use in Equation (5). The standard formula from the free electron model for the chemical potential for non-relativistic particles is given by

$$
\mu_{v 0}=\varepsilon_{F}=\left[(\hbar c)^{2} / 2 m_{b v} c^{2}\right]\left(3 \pi^{2} \rho\right)^{2 / 3},
$$

and relevant values are shown in Table 3.

One may set the chemical potential equal to the Fermi energy and use Equation (5) with these chemical potentials and with the associated upper limit $\left(2 m \varepsilon_{F}\right)^{1 / 2}$. The resulting densities at $T=155 \mathrm{~K}$ range from $72 \%$ to $60 \%$ of the results of Table 2 for the masses shown. The computed densities are not equal to the input densities because the integral does not converge with the aforementioned upper limit. For masses above $0.1 \mathrm{eV} / \mathrm{c}^{2}$, if the upper limit of the integral is extended until it converges, the resulting densities are consistent with the input densities from Table 2. This state of matter corresponds to Fermi temperatures varying from 162 to $172 \mathrm{~K}$, and to thermodynamic temperatures ( $T$ in Equation (5)) of 2 to $15 \mathrm{~K}$, with little variation in that range of $T$. The lower thermodynamic temperatures are expected from the theory of metals. For masses at or below $0.1 \mathrm{eV} / \mathrm{c}^{2}$, the particles are sufficiently relativistic that the Fermi temperatures need to be adjusted to maintain consistency with Table 2. For these

Table 3. Fermi energy and Fermi temperature versus baryonic neutrino mass. Numbers in parenthesis are adjusted values to account for relativistic effects at lower masses, to match densities of Table 2.

\begin{tabular}{cccccccc}
\hline \multicolumn{7}{c}{ Mass $\left(\mathrm{eV} / \mathrm{c}^{2}\right)$} \\
\hline & 0.025 & 0.05 & 0.1 & 0.2 & 0.3 & 0.4 & 0.6 \\
\hline Fermi Energy $\mathcal{E}_{F}(\mathrm{eV})$ & $\begin{array}{c}0.0241 \\
(0.018)\end{array}$ & $\begin{array}{c}0.0188 \\
(0.016)\end{array}$ & $\begin{array}{c}0.0161 \\
(0.015)\end{array}$ & 0.0148 & 0.0144 & 0.0142 & 0.140 \\
Fermi Temperature $T_{F}(\mathrm{~K})$ & 279 & 218 & 187 & & & & \\
& $(206)$ & $(187)$ & $(174)$ & 172 & 167 & 164 & 162 \\
\hline
\end{tabular}


lower masses, the Fermi temperature is set to 206,187 , and $174 \mathrm{~K}$ for masses of $0.025,0.05$, and $0.1 \mathrm{eV} / \mathrm{c}^{2}$, respectively. The result is that a thermodynamic temperature of 2 to $15 \mathrm{~K}$ still gives the densities of Table 2 for all masses shown. Hence $T$ will be set to $2 \mathrm{~K}$ in what follows, roughly consistent with the expected temperature of free neutrinos in the modern universe [12], p. 154. Given that the baryonic neutrinos were bound early in the history of universe when the mean kinetic energy was approximately $0.02 \mathrm{eV}$, the haloes comprising such matter should remain relatively stable after formation, much as bound ordinary matter. This expectation for halo evolution is justified in detail in Section 6 .

It is also possible that multiple species of baryonic neutrinos could result in lower average temperatures and lower RMS velocities that further reduce diffusion of the hypothesized matter away from galactic centers. There are 3 basic types of neutrinos, so there are expected to be at most $3^{3}=27$ possible types of a colorless baryonic triplet, just as with the discrete $\mathrm{SU}(3)$ symmetry for $(u, d, s)$ states in the quark sector. Accounting for antiparticles there may be as many as 54 species. To achieve the same total number density, one then requires a temperature of $12 \mathrm{~K}$ with 54 species rather than $155 \mathrm{~K}$, assuming a Fermi-Dirac distribution and a mass of $0.4 \mathrm{eV} / \mathrm{c}^{2}$. The RMS velocity in such cases is about $0.09 \mathrm{c}$, which is still sufficient to overcome a galactic escape velocity if there is no other form of binding. One might expect that all such species would be present in the hot early universe. Then, as the universe cools only a few species that are most stable would remain, matching the known behavior of the quark sector. With this in mind the analyses related to the modern era will assume one or two stable baryonic neutrino species. It is possible that a few mesonic neutrino states may be present. The net effect of two baryonic neutrino states would change the Fermi temperature from $155 \mathrm{~K}$, for example, to about $99 \mathrm{~K}$ to achieve the same total density (at a mass of $0.4 \mathrm{eV} / \mathrm{c}^{2}$ ).

The mid and upper range of energy densities in Table 2 is somewhat greater than the current nominal estimate of $0.3 \mathrm{GeV} \cdot \mathrm{cm}^{-3}$ for the dark matter mass-energy near earth [41] [59] [60], which is a desirable property for dark matter near a galactic center.

Next, the distribution of ordinary matter must be specified. In recent years, the model of choice [47] for describing the projected density of elliptical galaxies is due to Sérsic [48]. An approximate de-projected form is given by [61]. A similar form for the density versus radius was developed by Einasto [49]. The center bulge of the Milky Way has a cluster with a Sersic exponent of $n=3$ [50] for OM. As is well known, for spiral galaxies the overall Sersic exponent $n$ is a measure of the balance between the disk and the bulge, two clearly distinct components. A typical Sersic exponent for a spiral galaxy might be 4 or more, but for the central bulge 2 is a common number [51]. For elliptical galaxies, $2-4$ is a common number [42] for OM. With these various results in mind, a Sersic exponent of 2 is initially chosen for ordinary matter. The projected form of the Sersic equation is

$$
\rho_{\text {rad }}=\rho_{\text {rad } 0} \exp \left(-A r^{a}\right)
$$

where $\rho_{\text {rado }}, A$, and $\alpha$ are constants. The de-projected Sersic (dpS) density distri- 
bution for radiant matter is approximated by [42] [61]:

$$
\rho_{\text {rad }}=\rho_{\text {rad } 0}\left(r / R_{e}\right)^{-p_{n}} \exp \left[-b_{n}\left(r / R_{e}\right)^{1 / n}\right] \text {. }
$$

The parameter $\rho_{\text {rad } 0}$ is obtained by setting the volume integral of Equation (8) equal to the measured or inferred ordinary mass of the galaxy. The variable $R_{e}$ denotes the radius which encloses $1 / 2$ the total light of the galaxy. The other two parameters in Equation (8) are given conveniently and approximately from Equation (19) and Equation (27) of [42],

$$
p_{n}=1.0-0.6097 / n+0.05463 / n^{2} \text {, }
$$

and

$$
b_{n}=2 n-1 / 3+0.009876 / n \text {. }
$$

Figure 1 shows the $n=2 \mathrm{dpS}$ radial profile and also a profile with $n=4$. The assumed total OM mass of the galaxy is assumed to be $M_{g a l}=9 \times 10^{10}$ solar masses, approximating that of the Milky Way [60] [62]. It is assumed to have a black hole of $4 \times 10^{6}$ solar masses at its center.

The dpS profile is also used with some success for characterization of the DM density profile versus radius. Another density distribution used for characterization of DM is the Einasto distribution, which for the purposes of this paper is given by

$$
\rho_{\text {Ein }}=\rho_{0} \exp \left[-d_{n}\left(r / R_{e, \mathrm{DM}}\right)^{1 / n}\right],
$$

where $R_{e, \mathrm{DM}}$ is the radius of the volume enclosing $1 / 2$ the total of the galaxy, and $d_{n}$ is given approximately by Equation (24) of [42]:

$$
d_{n} \approx 3 n-1 / 3+0.0079 / n \text {, for } n>0.5 \text {. }
$$

Both the dpS and Einasto distributions will be compared against the DM density profiles computed using Equation (1) through (5).

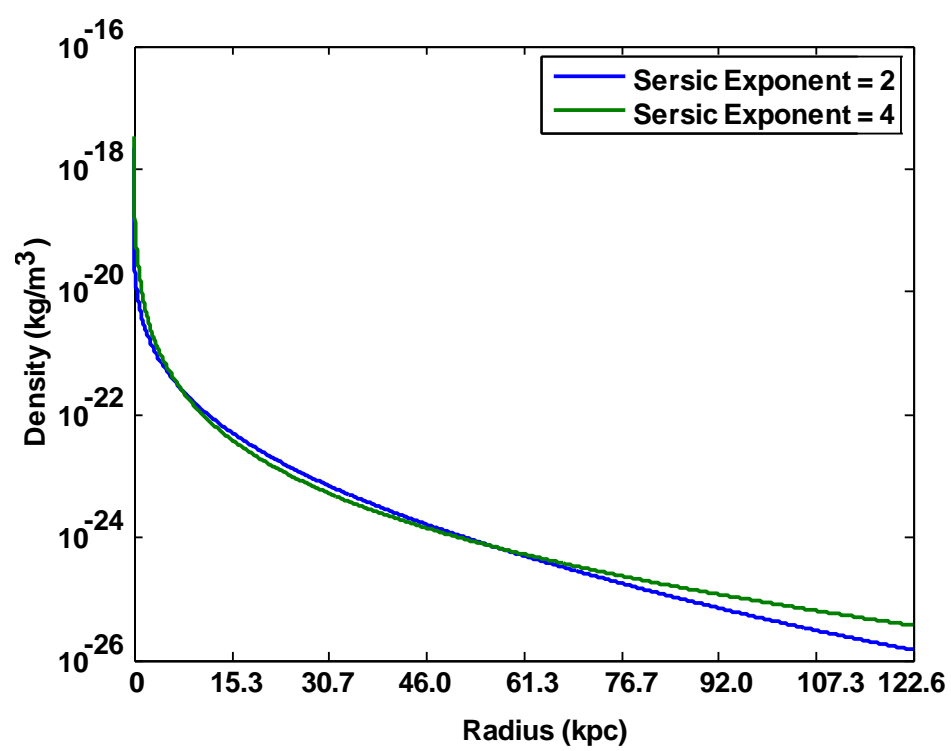

Figure 1. Profiles for a de-projected Sersic model with Sersic index of 2 (blue) and 4 (green). Radius parameter $=15.3 \mathrm{kpc}$. 
The radiant matter distribution is the source term for Equation (1) and Equation (2). To use these equations to compute a profile, a polytropic exponent must be chosen. Based on the discussions above, two polytropic exponents are considered, $\gamma=5 / 3$ and $\gamma=2-\varepsilon$, with $\varepsilon$ a positive number much less than 1. The polytropic relation for $\gamma=5 / 3$ is given above. For the case of $\gamma=2-\varepsilon$, one can use the polytropic relation $P=c_{2-\varepsilon} \rho^{2-\varepsilon}$ with $c_{2-\varepsilon}$ given by [55]

$$
c_{2-\varepsilon}=(2 / \pi) G m_{b v}^{2} R_{g h}^{2} \rho_{0}^{\varepsilon} \equiv P_{0} / \rho_{0}^{2-\varepsilon} .
$$

Here $R_{g h}$ is the nominal radius of the galactic halo, chosen to be $92 \mathrm{kpc}$ for the purposes of this paper. It will be checked for consistency in the following solutions. The pressure $P_{0}$ at the center of a galactic halo is defined by Equation (10). So, for example, with a baryonic neutrino mass $m_{b v}$ of $0.4 \mathrm{eV} / \mathrm{c}^{2}$ and Equation (11) one obtains $c_{2}=2.08 \times 10^{-40} \mathrm{~J} \cdot \mathrm{m}^{3}$. In this case, $P_{0}=1.53 \times 10^{-9} \mathrm{~J} \cdot \mathrm{m}^{-3}$. For $\rho$ in units of $\mathrm{m}^{-3}$ one obtains the correct units in Equation (11). Other approaches are available to estimate the value of $c_{2-\varepsilon}$, for example as given in [52]. Such an approach gives a value within 1.5 orders of magnitude of that given here.

This section provided the equations and the input parameters needed to compute solutions for DM density. Variations about these input assumptions are also considered.

\subsection{Galactic-Scale Solutions}

Moving on to full numerical solutions of Equations (1)-(5), consider conditions corresponding to a galaxy similar to the Milky Way as mentioned above. To obtain a solution, a density at the origin must be specified. These are given in the second column of Table 2 for the respective masses. For example, for a baryonic neutrino mass of $0.4 \mathrm{eV} / \mathrm{c}^{2}$, the number density is $\rho_{0}=5.34 \times 10^{15} \mathrm{~m}^{-3}$.

Table 4 summarizes the inputs used for the results of this section. Note that the quoted temperature is the thermodynamic temperature used in Equation (5), with chemical potentials $\mu_{\nu}$ given in Table 3 .

Table 4. Inputs for calculations of this section.

\begin{tabular}{|c|c|c|}
\hline Input Parameter & Values & Comment \\
\hline Thermodynamic Temperature, $T$ & $2 \mathrm{~K}$ & Tof Equation (5) \\
\hline Fermi Temperature $T_{0}$ at origin & $160-210 \mathrm{~K}$ & From Table 3 \\
\hline Masses of baryonic neutrinos, $m_{b v}$ & $.025-0.6 \mathrm{eV} / \mathrm{c}^{2}$ & Upper limit of mass is from Appendix A \\
\hline Galactic halo radius, $r_{c}$ & $92 \mathrm{kpc}$ & $r_{c}$ in Equation $(4)$ \\
\hline Polytropic exponents & $5 / 3$ to 2 & As discussed above \\
\hline Ordinary matter profile & dpS profile & Exponent $=2$, radius $=15.3 \mathrm{kpc}$ \\
\hline Total ordinary matter mass, $M_{g a l}$ & $9 \times 10^{10}$ & Solar masses \\
\hline $\begin{array}{l}\text { Fermi Temperature } T_{o u t} \text { at radius } r_{\mathcal{o}} \\
\text { with chemical potential } \mu_{v c} \equiv k T_{\text {out }}\end{array}$ & $5-16 \mathrm{~K}$ & $\begin{array}{l}\text { Sets density } \rho_{c} \text { at outer radius in } \\
\text { Equation (4) using Equation (5) }\end{array}$ \\
\hline Inner scale $r_{0}$ & $1 \mathrm{kpc}$ & $\begin{array}{l}\text { Sets radius of constant density region in } \\
\text { Equation (4), approximate size of bulge of } \\
\text { Milky Way }\end{array}$ \\
\hline
\end{tabular}


The numerical integration of Equation (1) and Equation (4) for the density uses 4000 steps at $30.7 \mathrm{pc}$ each. A simple finite-difference numerical approach proves adequate with the quoted step size. If the density is reduced below $10^{9} \mathrm{~m}^{-3}$, then the density is set to that value for display purposes.

The results of the calculations are shown in Figure 2 and Figure 3 for the standard and generalized equations of hydrostatic equilibrium, respectively. The horizontal axes in these figures are the dimensionless ratio radius/ $R_{g h}$, where $R_{g h}$ $=92 \mathrm{kpc}$ as discussed above. A comprehensive (but not exhaustive) search was performed over fermionic mass, polytropic exponent, and mean kinetic energy within the ranges shown in Table 4 . The search attempted to find the best match to the following properties of dark matter reported in the literature: 1 ) outer radius
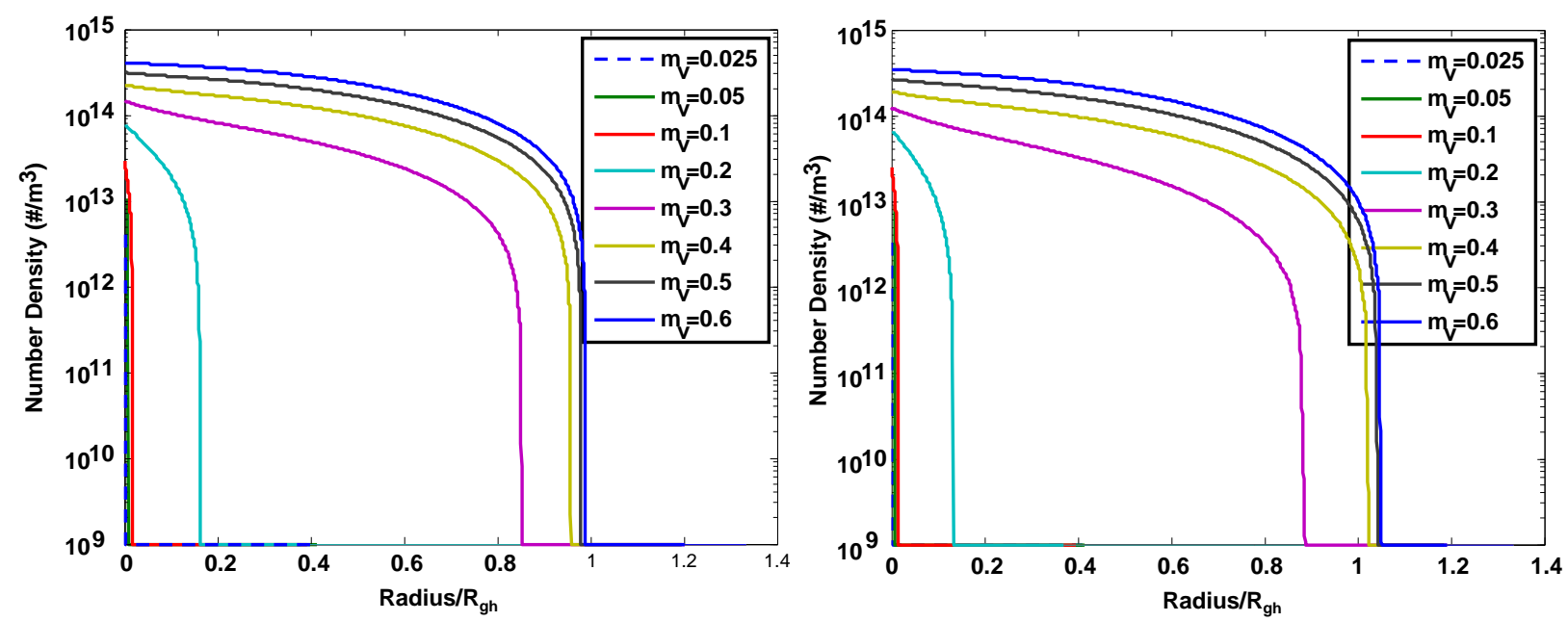

Figure 2. Calculated dark-matter profiles versus radius for various masses of a baryonic neutrino for a galaxy similar to the Milky Way with a Sersic index of 2. Standard hydrostatic equation assumed. Legend shows masses ranging from 0.025 to 0.6 eV/c ${ }^{2}$. Left: $\gamma=1.995, T_{0}=19$ to $20 \mathrm{~K}$. Right: $\gamma=1.90, T_{0}=17 \mathrm{~K}$.
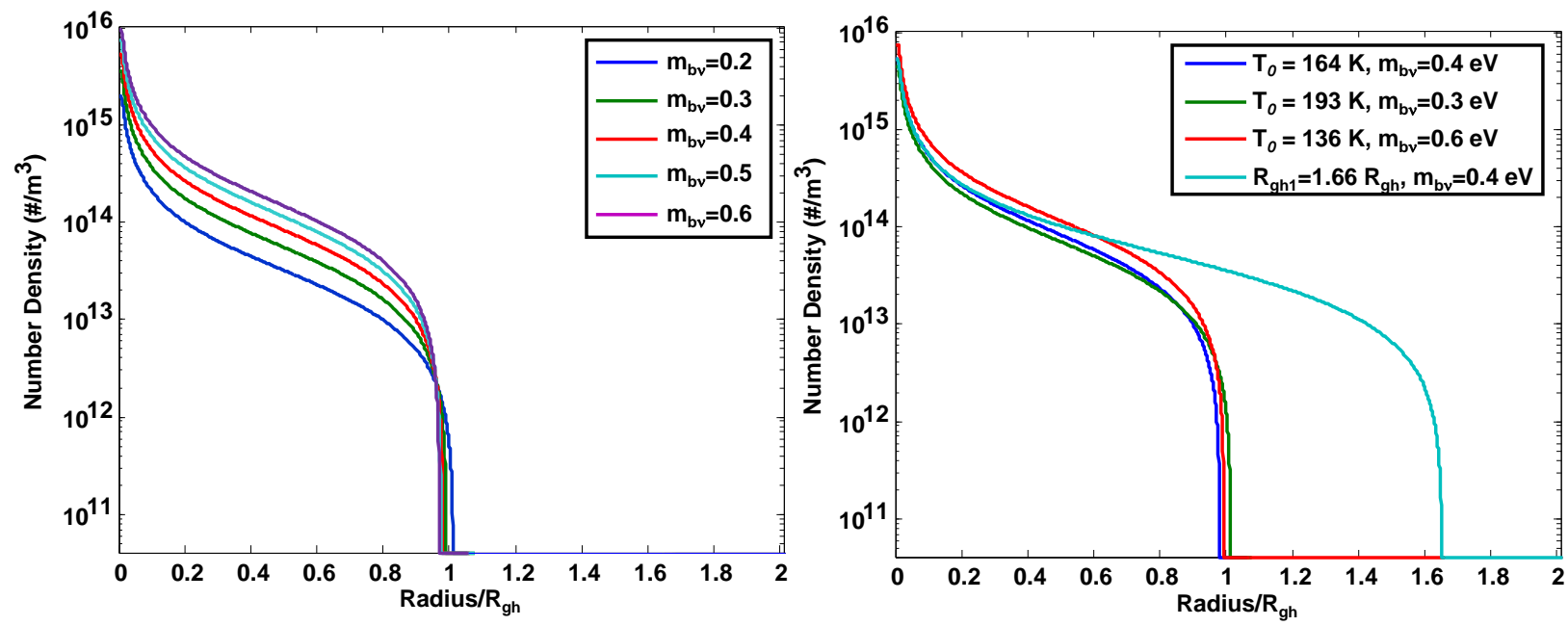

Figure 3. Calculated dark-matter profiles versus normalized radius for various masses of baryonic neutrino for a galaxy similar to the Milky Way. Generalized hydrostatic equation assumed. Left: varying mass, $T_{0}$ as shown in Table $4, r_{0}=1 \mathrm{kpc}, T_{\text {out }}$ $=8 \mathrm{~K}$. Right: $T_{0}$ varied as given in the legend to overlay curves for different masses. Also shown is a case with galactic halo radius set to $1.66 R_{g h}=153 \mathrm{kpc}=r_{\mathcal{c}}$, with $m_{b v}=0.4 \mathrm{eV} / \mathrm{c}^{2}, T_{0}=155 \mathrm{~K}, r_{0}=1 \mathrm{kpc}$, and $T_{\text {out }}=5 \mathrm{~K}$. 
consistent with the assumed radius of DM of $92 \mathrm{kpc} ; 2)$ cusp near the origin; 3) mass-energy density at radius of Sol; 4) ratio of DM in galactic halo to OM in galaxy; 5) quantitative shape consistent with published simulations and inferences from observations, and 6) consistency of temperature at outer radius with standard cosmology. The best or near-best fits considering all these criteria are shown in the figures.

Results for the standard hydrostatic equation, Equation (1), are shown in Figure 2 for varying masses of baryonic neutrino and for two different polytropic exponents near 2 . The results for the cutoff radius shown here are a good match to the assumed radius of DM, $R_{g h}=92 \mathrm{kpc}$, for particle masses ranging from 0.3 to $0.6 \mathrm{eV} / \mathrm{c}^{2}$. However, as expected, it is seen that the results using the standard hydrostatic equation are not a good match to a dpS profile, by comparison to Figure 1. No cusp is present. The quantitative shape is also not a match to any accepted dpS profiles. The shapes shown in Figure 2 are typically close to the expected result for the Lane-Emden equation, $\sin (\pi r / R) /(\pi r / R)$ for some $R$, assuming the standard hydrostatic equation with a polytropic exponent near 2 . The shapes are expected to differ somewhat from this standard form because (a) the exponents are not exactly 2, and (b) the solutions shown are for the inhomogeneous form of the equation with a strong concentration of $\mathrm{OM}$ at and near the origin.

It should also be noted that with a polytropic exponent near 2 and using Equation (11), the lower masses have less pressure and so result in smaller half-max radii. This differs from the case of a polytropic exponent of $5 / 3$, which yields larger half-max radii for lower masses in view of the expression for fermions given after Equation (5). With smaller exponents such as 5/3, dramatically larger galactic haloes are computed using the standard hydrostatic equation, and so are not shown.

Several other metrics are worth discussion for Figure 2. First, the total enclosed mass is in the range of 4 to 60 times the ordinary matter for particle masses ranging from 0.3 to $0.5 \mathrm{eV} / \mathrm{c}^{2}$ for the plots shown. For $0.4 \mathrm{eV} / \mathrm{c}^{2}$, the total enclosed mass is within $15 \%$ of 15 times ordinary matter. Second, the mass-energy density (as computed in Table 2) at the radius of Sol is 0.06 to $0.2 \mathrm{GeV} \cdot \mathrm{cm}^{-3}$ for particle masses ranging from 0.4 to $0.6 \mathrm{eV} / \mathrm{c}^{2}$ for Figure 2. Values of $T_{0}$ much larger or smaller than the range shown in Figure 2 do not show a radius consistent with the assumed halo radius for $\gamma$ near 2. Overall, the results of Figure 2 are a match to the assumed halo radius and enclosed mass for some particle masses. However, such solutions have a relatively low energy density at earth compared to observational inferences and also do not have the cusp deduced from data.

Sample results for the generalized hydrostatic equation, Equation (4), are shown in Figure 3. In this case, as in Figure 2, the plots with the specified inputs also show a radius that is in the neighborhood of $R_{g h}=92 \mathrm{kpc}$, consistent with the assumed radius. However, it is seen that the results using the generalized hydrostatic equation are a qualitative match to a $\mathrm{dpS}$ profile. A cusp is present out- 
side of a radius of $1 \mathrm{kpc}$ with a logarithmic slope of about -1 .

The left plot of Figure 3 shows the sensitivity of the result to the mass of the hypothesized baryonic neutrino. It is seen that the range of masses that are consistent within $10 \%$ of $R_{g h}$ is from 0.2 to $0.6 \mathrm{eV} / \mathrm{c}^{2}$ for the chosen inputs. Lower masses did not show such agreement. The right plot shows sensitivity to $T_{0}$ in the vicinity of the nominal $155 \mathrm{~K}$, showing the masses required to obtain a similar density profile. The right plot also shows a sample case for the galactic radius set to $1.66 R_{g h}=153 \mathrm{kpc}$ with a baryonic neutrino mass of $0.4 \mathrm{eV} / \mathrm{c}^{2}$ with appropriate choice of temperatures. The point of this plot is that other (self-consistent) galactic halo radii can be achieved with similar input parameters.

Further calculations, not shown, give consistent results for $R_{g h}$ with particle masses up to about $5.0 \mathrm{eV} / \mathrm{c}^{2}$ for smaller values of $r_{0}, r_{0}$ as low as $0.1 \mathrm{kpc}$. These typically require lower values of $T_{0}$ and $T_{\text {out }}$. For larger particle masses, the temperature needed for $T_{\text {out }}$ is $0.1 \mathrm{~K}$ or less to match both galactic halo size $\left(R_{g h}\right)$ and enclosed DM mass. For larger values of particle mass, above $5.0 \mathrm{eV} / \mathrm{c}^{2}$, consistency with both observationally-inferred galactic halo size and mass cannot be met simultaneously; either the computed radius matches $R_{g h}$ but the total enclosed DM mass is too large, or the total mass matches expectations but the radius is too small. Dwarf galaxies with masses of $10^{7}$ to $10^{10}$ solar masses can be obtained with this approach for particle masses of $0.2 \mathrm{eV} / \mathrm{c}^{2}$ to $0.6 \mathrm{eV} / \mathrm{c}^{2}$. The required input values for dwarf galaxies are $T_{0}$ the same as in Table 4, with $r_{0}$ in the same range ( 0.1 to $1 \mathrm{kpc}$ ), $R_{g h}=1$ to $2.5 \mathrm{kpc}$, and $T_{\text {out }}=30$ to $95 \mathrm{~K}$.

Figure 4 shows plots of two relevant metrics. The curve on the left of Figure 4 shows the ratio of the enclosed DM mass to OM mass for the curves on the left of Figure 3. A dotted line shows the estimated universal average ratio of $6: 1$. This ratio is believed to be of the order of 15:1 for the galactic halo of the Milky Way [60]. The values of baryonic masses consistent with ratios of 6:1 to 30:1 are about 0.25 to $0.50 \mathrm{eV} / \mathrm{c}^{2}$.
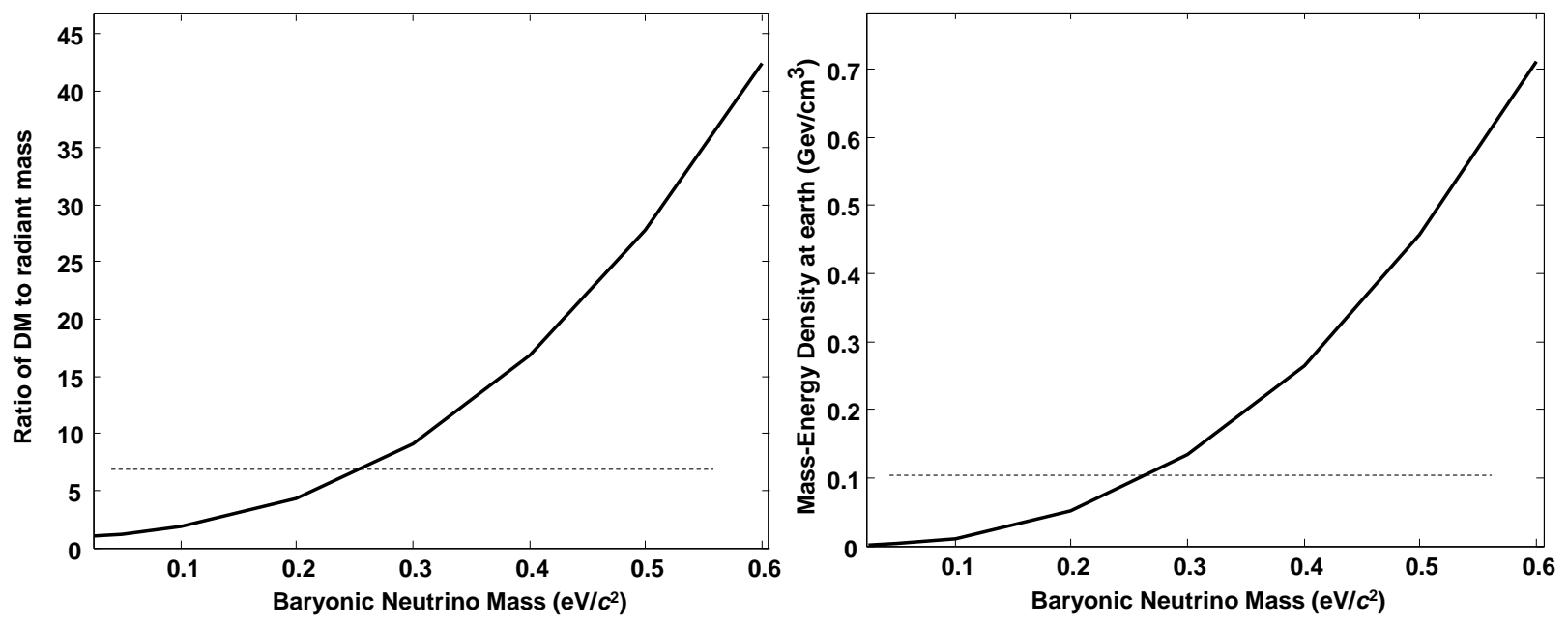

Figure 4. Left: Computed ratio of $\mathrm{DM}$ to $\mathrm{OM}$ versus mass of the baryonic neutrino $\left(\mathrm{eV} / \mathrm{c}^{2}\right)$ for conditions corresponding to the left plot of Figure 3. Right: Computed mass-energy density of DM at the radius of earth for conditions corresponding to the left plot of Figure 3. 
The plot on the right of Figure 4 shows the computed mass-energy density of $\mathrm{DM}$ at a radius of $7.9 \mathrm{kpc}$ for plots on the left of Figure $3.7 .9 \mathrm{kpc}$ corresponds to the distance of earth from the galactic center. As discussed above, the mass-energy density is given by $\rho m_{b \nu} c^{2} /\left[1-\left(v_{r m s} / c\right)^{2}\right]^{1 / 2}$. As mentioned earlier, the estimated mass-energy density at earth is $0.3 \mathrm{GeV} \cdot \mathrm{cm}^{-3}$, "within a factor of $2-3$," as stated in the Astrophysical Constants section of the most recent PDG document [41]. The resulting lower bound is $0.1 \mathrm{GeV} \cdot \mathrm{cm}^{-3}$, and this lower bound is shown in the right plot as a dotted line. The particle masses consistent with these estimates from data are about 0.25 to $0.65 \mathrm{eV} / \mathrm{c}^{2}$.

The nominal computed density profile from Figure 3 with a baryonic neutrino mass of $0.40 \mathrm{eV} / \mathrm{c}^{2}$ is fit to various model profiles as shown in Figure 5. In addition to this single-species computation, a two-species computation is also shown, and will be discussed below. The densities of Figure 5 are all limited from above to the value of $m_{b v} \rho_{0}=3.76 \times 10^{-21} \mathrm{~kg} \cdot \mathrm{m}^{-3}$ within $1 \mathrm{kpc}$ of the origin, consistent with the nominal computed profile for a baryonic neutrino mass of $0.40 \mathrm{eV} / \mathrm{c}^{2}$. The vertical axis is normalized by this density. This limit at the origin is used and shown because it is in better accord with most measured data [63] [64] [65] than the model profiles and also because such a limit is in better accord with a density that is limited by Fermi-Dirac statistics. Just outside this core, all densities of all profiles of Figure 5 have an approximate $1 / r$ dependence, including the dpS and Einasto model profiles. However, at larger radii, the nominal density profile has a slower decline than the dpS and Einasto model profiles up to a radius of about $0.9 R_{g h}$.

The chosen parameters for the model profiles are given in Table 5. The parameters for the dpS and Einasto models are within the accepted range for the exponent and the radius, by comparison with the values shown at the bottom of

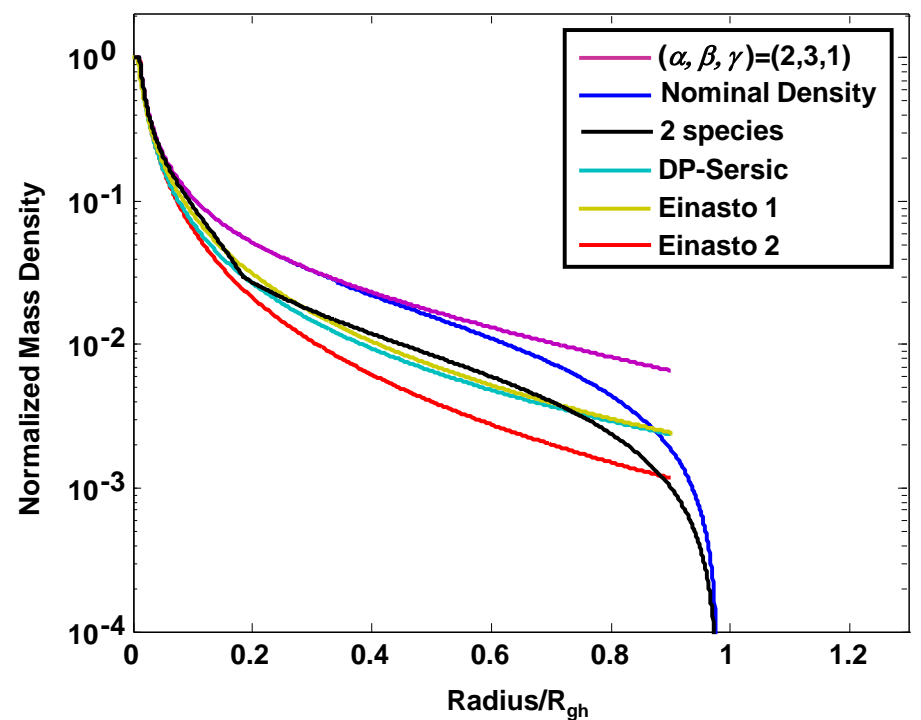

Figure 5. Nominal computed density profile of Figure 3 with a baryonic neutrino mass of $0.40 \mathrm{eV} / \mathrm{c}^{2}$, a 2-species computed profile, and various model profiles. Model inputs and fit metrics are given in Table 5. 
Table 5. Model parameters and fit metrics of model profiles versus computed profiles for Figure $5^{\mathrm{a}}$.

\begin{tabular}{ccccccc}
\hline Input Parameters and Metrics: & $n$ & $R_{e, \mathrm{DM}}(\mathrm{kpc})$ & $\rho_{0} / \rho_{0, m V}$ & Metric 1 & Metric 2 & $M / M_{\text {gal }}$ \\
\hline $\begin{array}{c}\text { (2, 3, 1), fit to nominal } \\
\text { dpS, fit to nominal }\end{array}$ & - & $r_{02}=92$ & 1 & 0.20 & 0.91 & 19.6 \\
Einasto-2, fit to nominal & 5.3 & 700 & 0.011 & 0.47 & 0.45 & 7.8 \\
dpS, fit to 2-species & 3.3 & 400 & 220 & 0.61 & 0.68 & 4.9 \\
Einasto-1, fit to 2-species & 5.5 & 700 & 0.011 & 0.033 & 0.18 & 7.8 \\
Einasto-2, fit to 2-species & 5.5 & 800 & 110 & 0.011 & 0.20 & 8.6 \\
Galaxy-sized haloes, dpS & $3.1-4.6$ & $110-230$ & - & - & - & - \\
Galaxy-sized haloes, Einasto & $5.3-7.8$ & $190-400$ & - & - & - & - \\
Cluster-sized haloes, dpS & $2.2-3.5$ & $700-4700$ & - & - & - & - \\
Cluster-sized haloes, Einasto & $3.9-7.4$ & $1200-6000$ & - & - & - & - \\
\hline
\end{tabular}

${ }^{a}$ Bottom four rows are from [42].

the table, which are from [42]. However, as expected, the central density parameters are quite different. This is expected because of the large derivatives of the model profiles at the origin, as discussed in [52], which do not match measured values [63].

Table 5 also shows two metrics for the quality of the fits. These metrics are

$$
\text { Metric } 1=\left\{\Sigma_{r} U(r)\left[\rho_{M}(r)-\rho_{C}(r)\right]^{2} r^{2}\right\}^{1 / 2} /\left\{\Sigma_{r} U(r)\left[\rho_{C}(r)\right]^{2} r^{2}\right\}^{1 / 2}
$$

and

$$
\text { Metric } 2=\left\{\Sigma_{r} U(r)\left[\rho_{M}(r) / \rho_{C}(r)-1\right]^{2} r^{2}\right\}^{1 / 2} /\left\{\Sigma_{r} U(r) r^{2}\right\}^{1 / 2},
$$

where $\rho_{C}(r)$ is the numerically-computed profile and $\rho_{M}(r)$ is the model profile. Three classes of model profiles are considered: $(\alpha, \beta, \gamma)$, dpS, and Einasto. The metrics are computed over the range of radii for which the densities are appreciable $\left(U(r)=1\right.$ for $r<0.9 R_{g h}, 0$ otherwise). The metrics for these model fits to the nominal curve vary from $20 \%$ to $91 \%$, which is not particularly good, but are expected from visual inspection of Figure 5. It should be noted that both metrics 1 and 2 weight the agreement at the outer radii quite heavily, and this is a significant contributor, especially for the $(\alpha, \beta, \gamma)$ model.

Table 5 additionally shows the total enclosed mass within a radius of $0.9 R_{g h}$. For reference, the nominal density profile, the blue curve of Figure 5 has a total enclosed mass of $15.2 M_{g a l}$ within this radius. The $(\alpha, \beta, \gamma)$ model has an enclosed mass close to expectations, about $19 M_{g a l}$. The profiles labelled dpS and Einasto have a total enclosed mass ratio (DM/OM) that is low compared to expected values of 15 or more. Also considered but not shown are dpS and Einasto profiles with an inner core that is about $2 \mathrm{kpc}$ in radius, matching the mean radius of the bulge in the Milky Way. These had higher enclosed masses, of the order of 10 to $15 M_{\text {gal }}$ but the fit metrics were no better than shown in the table. 
Overall, the differences between the best-fit dpS and Einasto models and the nominal computed solution are not negligible. This difference can be addressed via several physically reasonable approaches. Such approaches include (a) inclusion of angular momentum in the computation, (b) an allowance for non-equilibrium profiles at larger radii, or (c) inclusion of multiple particle species. These three approaches are discussed briefly in the following three paragraphs. As shown in Figure 5, approach (c) was found to readily provide a good match to observationally-inferred profiles.

Angular momentum profiles in CDM haloes have undergone considerable study using N-body simulations of particles that interact by gravity alone. Such studies include [66] [67] [68] [69] [70]. However, the parametric relationship between the density profile and the angular momentum profile has not been considered at length. Further, such studies are of limited relevance because they focus on non-interacting particles rather than the Fermi fluid posited in this paper. Such studies find typical estimated velocities of the order of $300 \mathrm{~km} \cdot \mathrm{sec}^{-1}$ associated with angular momentum for the Milky Way [71]. For a DM particle mass of $0.4 \mathrm{eV} / \mathrm{c}^{2}$, such velocities correspond to kinetic energies of about $2 \times 10^{-7}$ $\mathrm{eV}$, which is much less than the estimated inter-particle binding energy of $\sim 0.02$ $\mathrm{eV}$ of the posited Fermi fluid. Hence, by analogy with other well-understood liquid or semi-solid celestial bodies, the primary impact of angular momentum would be distortion of the halo, with a limited impact on the density profile.

Approach (b) involves appeal to density profiles that do not strictly adhere to the $1 / r$ equilibrium profile at larger radii. Such non-equilibrium profiles are discussed in [34] and [52] and are attributed to the ongoing process of halo accretion and mergers. These profiles were considered in this effort but were found to differ from the model profiles even more than the nominal $1 / r$ profile. This is because if the model profile and the $1 / r$ profile agree at small radii and the computed non-equilibrium profiles are even flatter at intermediate radii then they are always above the $1 / r$ profile. From Figure 5, one can see that such a profile would offer worse agreement at larger radii However, with a larger particle mass, e.g. about $0.6 \mathrm{eV} / \mathrm{c}^{2}$, a sharper decline near the center could support a flatter profile at larger radii, as shown in the 2 -species profile of Figure 5.

Approach (c) was also considered and the result is shown in the black curve of Figure 5. It can be seen that this 2 -species model is a particularly good match to the dpS and Einasto-1 models. Both model curves have a radius in the range of 700 to $800 \mathrm{kpc}$, corresponding to small galactic clusters. This 2-species approach can be described succinctly using the notation developed for Equation (4) and Equation (5). The mass density profile involving two species is denoted by $\rho_{m, 2-\text {-species }}(r)$ and can be written

$$
\rho_{m, 2 \text {-species }}(r)=m_{1} \rho\left(r, T_{01}, r_{01}, T_{c 1}, r_{c 1}, m_{1}, \mu_{v 1}\right)+m_{2} \rho\left(r, T_{02}, r_{02}, T_{c 2}, r_{c 2}, m_{2}, \mu_{v 2}\right)
$$

Clearly, there are more parameters in Equation (13) that permit a better fit a model profile. Experimentation with the parameters indicated that values of $m_{1}$ of 0.3 to $0.5 \mathrm{eV} / \mathrm{c}^{2}$ led to a good fit at larger radii. Larger masses, $m_{2}=0.6$ to 0.8 
$\mathrm{eV} / \mathrm{c}^{2}$, lead to a better fit at smaller radii with a steeper mass-density slope, while still matching the total enclosed mass. Larger masses led to excessive total enclosed mass, and smaller masses led to insufficient total enclosed mass given the range of values for the chemical potentials and temperatures shown above. Note also that both species of masses lie within the range of values expected from Appendix A. Table 6 shows the inputs used to obtain the 2-species curve shown in Figure 5.

In summary, assuming the generalized hydrostatic Equation (4) and the posited baryonic neutrinos with masses of $0.4 \mathrm{eV} / \mathrm{c}^{2} \pm 50 \%$, the results are roughly consistent with the following observationally-inferred and simulated properties of galactic-scale or cluster-scale DM structure reported in the literature: 1) halo width consistent with the assumed radius of $92 \mathrm{kpc}$ for a galaxy similar to the Milky Way; 2) relatively flat density profiles within a core radius of $\sim 1 \mathrm{kpc} ; 3$ ) cusp in the region outside of this core; 4) mass-energy density at radius of Sol; 5) ratio of DM in galactic halo to OM in a galaxy similar to the Milky Way; 6) qualitative shape; and with multiple species, 7) quantitative shape. Further, the temperature at the edge of the galactic halo is consistent with expectations from standard cosmology, with a temperature of $2 \mathrm{~K}$ (Fermi temperatures of 5 to 16 $\mathrm{K})$.

The above represents a summary of a search over multiple parameters, including baryonic-neutrino mass, particle temperature, polytropic exponents, and core radius. The standard and generalized hydrostatic equations are both considered. The generalized hydrostatic equation gives a better overall match for metrics derived from fits to representative models and data than does the standard equation for these ranges of values, when all 7 of the criteria mentioned in the previous paragraph are considered. However, the generalized hydrostatic equation of equilibrium does not fully trace to DM material properties. Only four of the six inputs to the solution are traceable to material properties: the particle mass and Fermi temperature of the constituent particle, both at the origin and outer radius. The other two inputs, the inner radius $r_{0}$ and the outer radius $r_{c}$ are not traceable to fundamental physical properties and make it too easy to fit some of the measured parameters. Nonetheless, selection of these two parameters allows a simultaneous fit to multiple criteria, which seems more than fortuitous.

\section{The Hypothesis and the Tremaine-Gunn Bound}

Reference [72] identified a bound that assumes (a) that the subject particles are fermions, (b) that such particles are non-relativistic, and (c) that such particles

Table 6. Parameters used for 2-species plot of Figure 5.

\begin{tabular}{ccccccc}
\hline Input Parameters & $m\left(\mathrm{eV} / \mathrm{c}^{2}\right)$ & $T_{0}=T_{c}(\mathrm{~K})$ & $r_{0}(\mathrm{kpc})$ & $r_{c}(\mathrm{kpc})$ & $\mu_{10} / k(\mathrm{~K})$ & $\mu_{v d} / k(\mathrm{~K})$ \\
\hline Species 1 & 0.4 & 2 & 1 & 92 & 104 & 8 \\
Species 2 & 0.6 & 2 & 1 & 9.2 & 51 & 16 \\
\hline
\end{tabular}


are non-interacting. In this paper, (a) is true, (b) is approximately true, but (c) is not true near a galactic center. Regarding (a), neutrinos should form baryonic neutrinos with odd multiples of half-integer spin, as expected by analogy with quark-based baryons. Regarding (b), the estimated speed of baryonic neutrinos for a temperature of $2 \mathrm{~K}$ is about $0.03 c$ to $0.05 c$ depending on mass, and possibly less if there are multiple species. Regarding $(c)$, the hypothesized baryonic neutrinos are weakly bound near a galactic center, based on the findings of Sections 3.1 and 3.2, and therefore interact. Hence (c) is not true. In the depths of space, far from any ordinary matter clusters, one might expect that neutrinos or the bound neutrino states considered herein are likely non-relativistic and not interacting and the Tremaine-Gunn bound is indeed expected to apply.

\section{The Hypothesis and Observation of DM near Earth}

One might question whether such baryonic neutrinos might be observed at earth. As seen in Figure 4, one finds consistency of the hypothesis for the mass-energy density near earth with accepted values [41] [59] for baryonic neutrino masses of 0.25 to $0.6 \mathrm{eV} / \mathrm{c}^{2}$. This basic comparison supports the hypothesis. Somewhat larger masses are possible as well.

It is estimated that the flux of solar neutrinos from the sun at earth is about 7 $\times 10^{10} \mathrm{~cm}^{-2} \cdot \mathrm{sec}^{-1}$, see, e.g. [73]. Given that solar neutrinos travel at nearly the speed of light, the corresponding density is about $2.3 \mathrm{~cm}^{-3}$. From the previous two sections, with the 2018 PDG mass-energy density of DM at the earth of $\sim 0.3$ $\mathrm{GeV} \cdot \mathrm{cm}^{-3}$ one obtains a baryonic neutrino density of about $10^{9} \mathrm{~cm}^{-3}$, assuming a baryonic neutrino mass of $0.3 \mathrm{eV} / \mathrm{c}^{2}$. Based on a simple scaling of the electroweak force which goes as the square of the CM energy, DM baryonic neutrinos should interact much more weakly than solar neutrinos via the electroweak force by a factor of about $1 /\left(3 \times 10^{5}\right)^{2}$ or less, because they have much lower energy than solar neutrinos in the earth reference frame $(<1 \mathrm{eV}$ for the former versus $\sim 0.3 \mathrm{MeV}$ for the latter). Hence, direct observation of such baryonic neutrinos seems challenging.

Further, solar neutrinos should interact in a very limited way with such DM via $\mathrm{SU}(3)_{v e}$, because solar neutrinos are predominately electron neutrino states, which are mono-color based on the theory of [38]. It should be noted that this property would not follow from a simple application of SU(3) to neutrinos. Specifically, they are color green using the conventions of that work. DM consists of baryonic states which are already colorless. Hence solar neutrinos cannot be absorbed or bound by DM in any significant way. Further, solar neutrinos cannot bind with each other to form baryonic or mesonic neutrinos to form colorless states since they are the same color (with no anti-color contribution). Thus, the interaction of solar neutrinos with themselves or DM should be limited to elastic (Rutherford) or quasielastic (Mott) scattering via SU(3) ${ }_{v}$. The latter would have similarities to high-energy electron scattering in bulk material. It will be seen in the companion paper that such elastic or quasielastic scattering should not sig- 
nificantly alter the composition, energy spectrum, or the flux of solar neutrinos as seen at earth, and this is consistent with observations to date. Note that solar neutrinos are not initially bound to other neutrinos partly because of the way they are created, but also partly because they are ultra-relativistic, just as quarks were not initially bound in the hot early universe. It should be re-stated that the experimental fact of neutrino oscillations is direct evidence that neutrinos will form bound states via $\mathrm{SU}(3)_{v e}$ within the context of the extended-color theory.

Possible observations for DM near earth also include an annual variation of the order of $1 \%$ of the time-average of scintillation in sodium iodide detectors at specific energies [74]. The observed energies of $\sim 6 \mathrm{keV}$ are not obviously related to the form of DM proposed herein. Measured values of the relative annual variation range from 0.0026 to 0.025 from Table 1 of that reference. The peak-to-valley velocity difference of earth relative to DM is about $v_{\mathrm{Sol}}=250 \mathrm{~km} \cdot \mathrm{sec}^{-1}$, i.e. $\sim 10^{-3} c$, assuming that DM is not rotating and the earth's orbital plane around the sun is oriented at $60^{\circ}$ relative to the sun's velocity vector around the galactic center [41]. The RMS velocity $V_{r m s}$ of the proposed DM is $0.03 c$ to $0.05 c$ with $T=2 \mathrm{~K}$. This gives an estimate of the relative annual variation of the flux of DM of $V_{\text {Sol }} / V_{r m s}=10^{-3} / 0.03$ to $10^{-3} / 0.05$. Thus, the estimated range of relative flux variation is a factor of 0.8 to 1.33 times the maximum measured relative variation of 0.025 quoted above.

The standard cosmological theory of neutrinos [12] [75] indicates that cold relic neutrinos are also present. The presence of bound neutrino states is not inconsistent with neutrinos interspersed with ordinary and DM matter. It is thus expected that there is a substantial fraction of free neutrino states near earth. Because of their low relative velocity, the helicity of such neutrinos of mass 0.005 to $0.055 \mathrm{eV} / \mathrm{c}^{2}$ would differ from those of high-energy free neutrinos observed in typical experiments, assuming that such neutrinos have mass and have Dirac wavefunctions. This would result in a relatively large fraction of right-handed neutrinos. Such neutrinos would be difficult to detect, however, because of the known inability to induce right-handed neutrinos to interact with normal matter [76]. Such relic neutrinos with a temperature of about $2 \mathrm{~K}$ would have electroweak cross sections comparable to those of bound neutrino states, and so would also be difficult to detect.

\section{The Hypothesis, Halo Stability, and Halo-Halo Interactions}

The hypothesis of SU(3) ${ }_{v}$ leads to an investigation of other self-interacting dark matter (SIDM) proposals. Recent papers [20] [21] [30] [31] discuss related phenomenologies. In the taxonomy of [21], this proposal is one of composite DM in which the mediators are both SU(3) massless gluons and/or lightly-broken SU(3) with massive mediators. In this case, the latter have mass of the order of the mass of the most massive constituent neutrino, and their effective binding energy is 4 to 12 times that of the highest-mass neutrino based on Appendix A.

With the self-interaction discussed here, DM is a dense form of matter that 
maintains its volume due to fermion degeneracy pressure of baryonic neutrinos. This dense form of matter is justified with the core assumption that the constituent baryonic neutrinos interact and are bound via $\mathrm{SU}(3)_{v}$ and obey non-relativistic Fermi-Dirac statistics. The $\mathrm{SU}(3)$ interaction with its approximate $1 / E^{2}$ dependence on CM energy $E$ [77] gives a large cross-section and a vanishing mean free path as temperatures tend to zero. Further, the computed Fermi temperature of Table 3 is typically much greater than the thermodynamic temperature of $2 \mathrm{~K}$, much as occurs with ordinary condensed matter. These considerations justify the simplest calculation for the mean free path, $\ell$ :

$$
\ell=\left(2^{1 / 2} \pi \rho d^{2}\right)^{-1}
$$

where $\rho$ is the density appropriate to the specific location in the halo and $d$ is the "size" of the particle. The values of $\rho$ are given by the densities of Table 2 assuming a macroscopic bound state with the given Fermi temperatures. The value of $d$ should be approximately $\hbar / p_{F}$, where $p_{F}$ is the Fermi momentum of the particle. The value of mean free path is shown in Table 7, along with the RMS velocity, $V_{r m s}$, which is $0.036 \mathrm{c}$ based on a temperature of $2 \mathrm{~K}$ and a particle mass of $0.4 \mathrm{eV} / \mathrm{c}^{2}$, in accord with the discussions following Table 3 . The table also shows the Fermi temperature, $T_{F}$ the diffusion constant, $\kappa=\ell v_{r m s}$, the mean free time between collisions, $\tau=\ell / v_{r m s}$, and the time to diffuse $1 \mathrm{kpc}, t_{1 \mathrm{kpc}}=(1 \mathrm{kpc})^{2} / \kappa$. Also shown is the medium pressure, assuming a polytropic exponent of 2 and assuming $c_{2}$ equals $2.08 \times 10^{-40} \mathrm{~J} \cdot \mathrm{m}^{3}$, as in the discussion surrounding Equation (11).

The table shows a diffusion constant varying from about 200 to $2000 \mathrm{~m}^{2} \cdot \mathrm{sec}^{-1}$, which is quite high compared to conventional matter. However, this diffusivity leads to negligible mass or heat transfer over scale sizes of the order of $1 \mathrm{kpc}$ over the age of the universe, as can be seen by the second-to-last column. This implies that the density and temperature distributions are expected to be relatively stable from the time of creation up to the present day, so that there is little evolution other than modest gravitational and SU(3)-based contraction and subhalo aggregation over most of the universe's lifetime. There are alternative formulations for the mean free path that differ from Equation (14), in which the cross section is calculated based on an interaction strength rather than the hard-sphere approximation. These estimates also lead to very long mass-transfer time constants, of the order of the age of the universe for haloes of $100 \mathrm{kpc}$ in size.

Table 7. Fluid properties of proposed DM versus density, assuming $m_{b V}=0.4 \mathrm{eV} / \mathrm{c}^{2}$ and temperature $T=2 \mathrm{~K}$.

\begin{tabular}{cccccccc}
\hline Density $\rho\left(\mathrm{m}^{-3}\right)$ & $T_{F}(\mathrm{~K})$ & $p_{F} / m_{b \nu} c$ & $I(\mu \mathrm{m})$ & $\kappa\left(\mathrm{m}^{2} \cdot \mathrm{sec}^{-1}\right)$ & $\tau(\mathrm{psec})$ & $t_{1 \mathrm{kpc}}(\mathrm{yrs})$ & $P\left(\mathrm{~J} \cdot \mathrm{m}^{-3}\right)$ \\
\hline $5 \times 10^{15}$ & 157 & 0.323 & 19.3 & 209 & 1.80 & $1.5 \times 10^{29}$ & $4.4 \times 10^{-9}$ \\
$5 \times 10^{14}$ & 34 & 0.148 & 40.9 & 442 & 3.79 & $6.9 \times 10^{28}$ & $4.4 \times 10^{-11}$ \\
$5 \times 10^{13}$ & 7.3 & 0.069 & 87.8 & 948 & 8.14 & $3.2 \times 10^{28}$ & $4.4 \times 10^{-13}$ \\
$5 \times 10^{12}$ & 1.6 & 0.032 & 189 & 2040 & 17.5 & $1.5 \times 10^{28}$ & $4.4 \times 10^{-15}$ \\
\hline
\end{tabular}


The above shows that the DM derived in this paper has some degree of self-consistency for a single halo. What does the above imply for halo-halo interactions? This is a very complex subject [34], involving baryonic feedback, dynamical friction, tidal stripping, and more. However, some calculations can be performed for the hypothesis under consideration. First, consider the kinetic energy of halo-halo interactions, which involve relative velocities $V_{\text {rel }}$ of 30 to $3000 \mathrm{~m} / \mathrm{sec}$, see e.g. [21]. The corresponding kinetic energy per particle $m_{b v} v_{r e l}^{2} / 2$ ranges from $2 \times 10^{-9}$ to $2 \times 10^{-5} \mathrm{eV}$. Note that these values are less than both the estimated inter-baryonic-neutrino binding energy, $6.4 \times 10^{-3} \mathrm{eV}$, as well as the estimated intra-baryonic neutrino binding energy, $0.4 \mathrm{eV}$. Hence the medium is not expected to dissociate, but rather to maintain its form.

Given that the medium is a fluid, the interaction should be characterizable in terms of dimensionless parameters such as Reynolds number, Re, and Mach number, Ma. These are shown in Table 8 for three interaction velocities and for the same 4 densities shown in Table 7. The Reynolds number is computed assuming the value of $\kappa$ in Table 7, the velocities shown, and a scale size of $1 \mathrm{kpc}$, which is assumed to be the typical diameter scale of a subhalo. The acoustic velocity is computed in the standard way assuming a polytropic exponent of 2 as in the discussion surrounding Equation (11).

From Table 8, it is evident that most of the Reynolds numbers are astronomical in comparison to typical values of terrestrial interest, and that the corresponding solutions are mathematically similar to those with very small viscosity. This, combined with the essentially incompressible properties of a quantum fluid satisfying Fermi-Dirac statistics, suggests that the fluid might approximate potential flow, which is known to have very low drag [78].

With such large Reynolds numbers, the accepted drag coefficient is about 0.2 for a sphere, as can be found in any textbook in fluid mechanics, neglecting possible quantum fluid effects. A drag coefficient as low as 0.1 occurs for an ellipsoid with a 2:1 aspect ratio, as may be found for subhaloes like the dwarf Sagittarius galaxy. With the above information, the drag-induced slowing of a subhalo in a larger medium can be computed using the simple differential relation

$$
\rho_{\text {sh }} \mathrm{d}|\boldsymbol{v}| / \mathrm{d} t V=\left(C_{d} / 2\right) \rho_{g a l}|v|^{2} A
$$

Here $\rho_{s h}$ is the DM density of the subhalo, set equal to $5 \times 10^{15} \mathrm{~m}^{-3}, \rho_{g a l}$ is the DM

Table 8. Reynolds number and Mach number, respectively, for halo-halo interactions. Scale size for $R e$ is $1 \mathrm{kpc}$, particle mass is $0.4 \mathrm{eV} / \mathrm{c}^{2}$.

\begin{tabular}{cccc}
\hline Density $\rho\left(\mathrm{m}^{-3}\right)$ & $V_{\text {rel }}=30 \mathrm{~km} \cdot \mathrm{sec}^{-1}$ & $V_{\text {rel }}=300 \mathrm{~km} \cdot \mathrm{sec}^{-1}$ & $V_{\text {rel }}=3000 \mathrm{~km} \cdot \mathrm{sec}^{-1}$ \\
\hline $5 \times 10^{15}$ & $4.4 \times 10^{21}, 0.019$ & $4.4 \times 10^{22}, 0.19$ & $4.4 \times 10^{23}, 1.9$ \\
$5 \times 10^{14}$ & $2.1 \times 10^{21}, 0.061$ & $2.1 \times 10^{22}, 0.61$ & $2.1 \times 10^{23}, 6.1$ \\
$5 \times 10^{13}$ & $9.8 \times 10^{20}, 0.19$ & $9.8 \times 10^{21}, 1.9$ & $9.8 \times 10^{22}, 19.1$ \\
$5 \times 10^{12}$ & $4.6 \times 10^{20}, 0.61$ & $4.6 \times 10^{21}, 6.1$ & $4.6 \times 10^{22}, 60.6$ \\
\hline
\end{tabular}


density of the galaxy in the vicinity of the subhalo, and $v$ is the velocity of the subhalo relative to the galaxy. $V$ is the volume of the subhalo, set equal to $4 \pi r_{s h}^{3} / 3$, and $r_{s h}$ is the subhalo radius, set equal to $0.5 \mathrm{kpc} . C_{d}$ is the drag coefficient, and $A$ is the cross-sectional area of the subhalo presented to the flow, set equal to $\pi r_{s h}^{2}$. Equation (15) is readily solvable assuming the densities are constant over time (as a first approximation). Sample results are shown in Table 9 versus initial relative velocity $V_{r e b}$ and local galaxy density, for times corresponding to $50 \%$ and $90 \%$ reductions in velocity.

The table shows, for example, that the 50\% and 90\% times are about 22 and about $197 \mathrm{Myr}$, respectively, for a subhalo diameter of $1 \mathrm{kpc}$ located at the outer edges of a galaxy where $V_{\text {re } 0}=300 \mathrm{~km} \cdot \mathrm{sec}^{-1}$. For haloes of order $1 \mathrm{kpc}$ in diameter and $v_{r e n}=30 \mathrm{~km} \cdot \mathrm{sec}^{-1}$, the orbit decay times range from about $0.2 \mathrm{Gyr}$ to $2 \mathrm{Gyr}$ based on the lower left values of Table 9. The lower end of this range, $0.2 \mathrm{Gyr}$, is definitely smaller than expected [34], for which typical subhalo decay times are predicted to lie in the range of 2 to 4 Gyr. This is a potential inconsistency which could be investigated via observations and simulations to test this theory as well as to inform the theory of the possibility of lower drag for this form of matter.

Referring to Equation (15), one sees that these numbers can be interpreted as the decay time per kpc diameter. So, for example, for a $10 \mathrm{kpc}$ subhalo such as the Sagittarius dwarf spheroid, the predicted velocity decay time constant is about $1970 \mathrm{Myr}$ for the $90 \%$ decay time with an initial velocity of $300 \mathrm{~km} \cdot \mathrm{sec}^{-1}$, referring to the bottom of the middle column of Table 9. If the shape of the subhalo is elliptical with major axis in the flow direction, as is the case for Sagittarius, the drag coefficient might be a factor of 2 lower, resulting a time constant of about 4 Gyr. These numbers are within a factor of 2 of those inferred from observations [79] [80]. It is not clear that this result is consistent with such observations, due to the neglect of many other effects such as the type of orbit and dynamical friction.

Another well-known constraint on self-interacting dark matter is the observation of the Bullet Cluster halo collision. In this collision the $r_{200}$ radius (the radius at which the galactic density is 200 times that of background) is about 2140 $\mathrm{kpc}$ for the main cluster and about $995 \mathrm{kpc}$ for the smaller Bullet Cluster. The final observationally-estimated lag of DM behind stellar matter is $25 \pm 29 \mathrm{kpc}$ [31] [81]. The drag-induced lag of the Bullet cluster halo relative to its associated ordinary stellar matter can be computed using Equation (15). Hernquist density

Table 9. Time (Myr) for velocity to decay by $50 \%$ and $90 \%$ for halo-subhalo interactions using Equation (15) and inputs described in text.

\begin{tabular}{cccc}
\hline Density $\rho_{\text {gal }}\left(\mathrm{m}^{-3}\right)$ & $V_{\text {reD }}=30 \mathrm{~km} \cdot \mathrm{sec}^{-1}$ & $V_{\text {rel }}=300 \mathrm{~km} \cdot \mathrm{sec}^{-1}$ & $V_{\text {reD }}=3000 \mathrm{~km} \cdot \mathrm{sec}^{-1}$ \\
\hline $5 \times 10^{15}$ & $0.22,1.97$ & $0.022,0.197$ & $0.002,0.020$ \\
$5 \times 10^{14}$ & $2.2,19.7$ & $0.22,1.97$ & $0.22,0.197$ \\
$5 \times 10^{13}$ & $21.8,197$ & $2.2,19.7$ & $0.22,1.97$ \\
$5 \times 10^{12}$ & 218,1970 & $21.8,197$ & $2.2,19.7$ \\
\hline
\end{tabular}


profiles are assumed as in [31] with the same initial velocity of $3000 \mathrm{~km} / \mathrm{sec}$ and same initial separation of $4 \mathrm{Mpc}$. Nominal motion is in a straight line. Values of closest approach of 10 to $100 \mathrm{kpc}$ of the cluster centers are used. A central number density of $5 \times 10^{15} \mathrm{~m}^{-3}$ at a radius of $1 \mathrm{kpc}$ is used for the main cluster, and number densities of $5 \times 10^{15}$ to $5 \times 10^{14} \mathrm{~m}^{-3}$ are used for central portion of the Bullet cluster, in accord with Section 3. The Bullet cluster radius is chosen to equal the Hernquist $\alpha$-parameter, $279 \mathrm{kpc}$, as in [31]. Using the $r_{200}$ radius of 995 kpc results in smaller lags due to the larger mass and therefore lower acceleration. A drag coefficient of 0.2 is used. The resulting computed lags range from 0.35 to $7.7 \mathrm{kpc}$, when $700 \mathrm{kpc}$ past closest approach, which is within error bars of that measured $(25 \mathrm{kpc})$.

Using a strict $1 / r$ density profile for the main cluster rather than the Hernquist profile gives a lag of as much as $15 \mathrm{kpc}$, because of the greater column density traversed by the Bullet Cluster. Also, a larger drag coefficient of 1.0 gives about 1.75 to $37 \mathrm{kpc}$ of lag instead of 0.35 to $7.7 \mathrm{kpc}$. If these larger numbers are combined with a $1 / r$ density profile, the result is about $73 \mathrm{kpc}$ of lag as an extreme worst case, which is outside the error bars. Convergence of these calculations was checked; the computed lags are accurate to within $0.01 \%$ using up to $10^{4}$ steps in time. The lags are relatively small because of the large size of the Bullet cluster halo, as noted in the previous paragraph, so its large mass decelerates less. Evidently, this form of self-interacting dark matter is consistent with the measured lag of the Bullet cluster collision, based on the simplest relevant drag calculation for this state of matter.

The net effect of the above calculations is decay of subhaloes into a larger halo, with associated erosion and assimilation. Scaling the results of Table 9, the slowing is most significant for the smaller haloes. From this, one sees a partial explanation for the unexplained dearth and diversity of smaller satellite haloes mentioned by other authors [21] [34]. Note that the decay rates for such haloes are significantly shorter than the time scales involving dynamical friction, so this offers observational means for assessing or informing this theory. Further, this picture of halo interaction is quantitatively consistent with the observed Bullet cluster halo interaction, provided the halo centers do not pass closer than about $10 \mathrm{kpc}$ of each other, using the simplest relevant calculation. The basic picture for halo-halo interactions is that a smaller dense halo moves through the less-dense perimeter of the larger halo in a manner similar to that of a mercury ball as it moves through water under the influence of gravity, with additional mass stripping due to friction and diffusion. Much more could be said about halo-halo interactions as it relates to the self-interacting form of DM that derives from the hypothesis of this paper; hopefully the above is sufficient for an initial treatment.

\section{Summary}

Straightforward calculations of galactic haloes are performed for DM assuming an $\mathrm{SU}(3)$ interaction applies to neutrinos. Both $\mathrm{SU}(3)_{v e}$ and $\mathrm{SU}(3)_{v s}$ are largely 
consistent with observations if one allows $\mathrm{SU}(3)_{v s}$ to have a different strength than that of $\mathrm{SU}(3)$ for quarks. The key attributes of this force can be and are chosen consistent with $\mathrm{SU}(3)_{v e}$ given by [38], whereas the standard SU(3) provides no guidance on key parameters such as the bound state mass or the coupling parameter, as discussed in Section 2.

As shown in Section 3, such baryonic neutrinos in haloes need a relatively high temperature to maintain the density and the associated total mass observed for haloes. Since the corresponding velocity exceeds the galactic gravitational escape velocity, some sort of additional binding is inferred. It is found that an $\mathrm{SU}(3)_{v}$ binding that is similar to the $\mathrm{SU}(3)$ binding of neutrons in neutron stars will produce sufficient binding. Because the resulting state of matter is similar to that for neutron stars, such haloes might be viewed as a form of "neutrino star". Such binding near galactic centers implies that the Tremaine-Gunn bound is not expected to apply as discussed in Section 4. The coupling constant $g_{s}$ of the feeble SU(3) ${ }_{v}$ is deduced to be as low as $\left[(137)^{2} \times 10^{-25}\right]^{1 / 4}=6.58 \times 10^{-6}$ of that of the electric force (e) from theoretical estimates. This coupling strength applies for relativistic neutrinos and is greater at lower energies due to the running of the coupling parameter as well as the interaction probability as discussed in Appendix A.

As shown in Section 3, a generalized form of the equation for hydrostatic equilibrium provides a better match than the standard equation for the observationally-inferred cuspy behavior for DM near galactic centers. Such calculations provide a good match to the inferred total galactic-halo mass and to the DM mass-energy density near earth. Solutions to the generalized hydrostatic equation are found to have long spatial "tails" that are cut off based on a galactic-halo mass constraint. Other explanations for the details of a halo density profile are explored. These include (a) multiple species, (b) angular momentum, and (c) a transition to a condition in which the standard hydrostatic equation applies. An approach using 2 species provides a particularly good fit to sample Einasto and de-projected Sersic model profiles. Further, the generalized solution offers a resolution to the "core-cusp" problem in dwarf galaxies. The solutions must have a core due to the density-limiting Fermi-Dirac statistics of baryonic neutrinos, in the absence of a gravitational singularity. Dwarf galaxies are found to have the "core" portion of the solution in Section 3.2, but either lose the cusp portion of their DM or never accumulate it.

Section 5 discusses the prospects for observation of such DM near earth. Solar neutrinos are not expected to interact significantly with each other or with such dark matter because they are created mono-color (all solar neutrinos are "green" in the conventions of [38]). Hence, they would not bind with each other or the colorless states of the hypothesized form of DM. However, elastic or quasi-elastic scattering might occur. Also, some contact is made with earth-based DM detection experiments, for which the measured amplitude of annual modulation is a fair match with what might be expected from this form of DM.

Halo solutions are stable and self-consistent, having low thermal and mass 
diffusivity as discussed in Section 6. Also shown in Section 6 is that the fluid hypothesis is consistent with observed galactic halo interactions (particularly the Bullet Cluster interaction) via arguments put forth here and by other authors, e.g. [82]. The issue of SIDM cross-section is apparently not relevant for the same reasons. Also considered is the motion of smaller haloes, less than $1 \mathrm{kpc}$ in diameter in a larger halo. It is found that the orbital decay is a factor of 1 to 10 times faster than expected from the standard DM model. Further observations of haloes and their interactions would provide helpful tests of the theory presented here. It remains to be seen if the hypothesis proposed herein has full consistency with the preponderance of observational evidence. Further work definitely remains.

A key issue for this form of dark matter is the free-streaming scale in the early universe. This issue is relegated to a companion paper. However, the calculations here show stability of haloes because of the relatively short mean free path of the hypothesized form of dark matter. This short mean free path applied to the early universe results in diffusive rather ballistic transport, vastly shortening the associated "free-streaming scale length" of such matter. Also covered in the companion paper is a discussion of the interaction strength of this form of DM with ordinary matter, dark-matter fraction of total matter, consistency with cosmic microwave background measurements, SN1987a data, neutrino accelerator anomalies, and the issue of $\mathrm{SU}(3)$ interactions between neutrinos and quarks

\section{Acknowledgements}

Portions of this work were presented in Paper APR19-000356 at the 2019 April Meeting of the American Physical Society. The material of this paper represents significant improvements over that of the conference paper. The author also wishes to thank T. Slatyer, and A. Nelson (deceased) for helpful discussions and E. Vishniac for critical comments on earlier versions of this paper.

\section{Conflicts of Interest}

The author declares no conflicts of interest regarding the publication of this paper.

\section{References}

[1] Doroshkevich, A.G., Khlopov, M.Yu., Sunyaev, R.A., Szalay, A.S. and Zeldovich, B.Ya. (1981) Annals of the New York Academy of Sciences 375, 32-42. https://doi.org/10.1111/j.1749-6632.1981.tb33688.x

[2] Bond, J.R., Centrella, J., Szalay, A.S. and Wilson, J.R. (1984) NATO Advanced Study Institute (ASI) Series C, 117, 87-99. https://doi.org/10.1007/978-94-009-7245-2_5

[3] Blumenthal, G.R., Pagels, H. and Primack, J.R. (1982) Nature, 299, 37-38. https://doi.org/10.1038/299037a0

[4] Bond, J.R., Szalay, A.S. and Turner, M.S. (1982) Physical Review Letters, 48, 1636-1639. https://doi.org/10.1103/PhysRevLett.48.1636

[5] Paczynski, B. (1986) The Astrophysical Journal, 304, 1-5. 
https://doi.org/10.1086/164140

[6] Griest, K. (1991) The Astrophysical Journal, 366, 412-421. https://doi.org/10.1086/169575

[7] Alcock, C., Allsman, R.A., Alves, D.R., Axelrod, T.S., Becker, A.C., et al. (2000) The Astrophysical Journal, 542, 281-307. https://doi.org/10.1086/309512

[8] Lee, B.W. and Weinberg S. (1977) Physical Review Letters, 39, 165-168. https://doi.org/10.1103/PhysRevLett.39.165

[9] Blumenthal, G.R., Faber, S.M., Primack, J.R. and Rees, M.J. (1984) Nature, 311, 517-525. https://doi.org/10.1038/311517a0

[10] White, S.D.M., Frenk, C.S. and Davis, M. (1983) The Astrophysical Journal, 274, L1-L5. https://doi.org/10.1086/184139

[11] Davis, M., Efstathiou, G., Frenk, C.S. and White, S.D.M. (1985) The Astrophysical Journal, 292, 371-394. https://doi.org/10.1086/163168

[12] Weinberg, S. (2008) Cosmology. Oxford University Press, Oxford.

[13] Liddle, A.R. (2004) Monthly Notices of the Royal Astronomical Society, 351, L49-L53. https://doi.org/10.1111/j.1365-2966.2004.08033.x

[14] Del Popolo, A. and Le Delliou, M. (2017) Galaxies, 5, Paper 17. https://doi.org/10.3390/galaxies5010017

[15] Pagels, H. and Primack, J.R. (1982) Physical Review Letters, 48, 224-227. https://doi.org/10.1103/PhysRevLett.48.223

[16] Covi, L., Kim, J.E. and Roszkowski, L. (1999) Physical Review Letters, 82, 4180-4183. https://doi.org/10.1103/PhysRevLett.82.4180

[17] Weinberg, S. (2013) Physical Review Letters, 110, Article ID: 241301. https://doi.org/10.1103/PhysRevLett.110.241301

[18] Dey, U.K., Ray, T.S. and Sarkar, U. (2018) Nuclear Physics B, 928, 258-267. https://doi.org/10.1016/j.nuclphysb.2017.12.005

[19] Allahverdi, R., Dutta, K. and Maharana, A. (2018) Journal of Cosmology and Astroparticle Physics, 18, 038. https://doi.org/10.1088/1475-7516/2018/10/038

[20] Robertson, A., Massey, R. and Eke, V. (2017) Monthly Notices of the Royal Astronomical Society, 467, 4719-4730. https://doi.org/10.1093/mnras/stx463

[21] Tulin, S. and Yu, H.-B. (2017) Physics Reports, 730, 1-58. https://doi.org/10.1016/j.physrep.2017.11.004

[22] Slatyer, T. (2018) Dark Matter: Where and What? SLAC Summer Institute Lecture, Stanford, August 2018.

[23] Hernquist, L., Katz, N., Weinberg, D.H. and Miralda-Escudé, J. (1996) The Astrophysical Journal, 457, L51-L55. https://doi.org/10.1086/309899

[24] Yoshida, N., Springel, V., White, S.D.M. and Tormen, G. (2000) The Astrophysical Journal, 544, L87-L90. https://doi.org/10.1086/317306

[25] Balberg, S., Shapiro, S.L. and Inagaki, S. (2002) The Astrophysical Journal, 568, 475-487. https://doi.org/10.1086/339038

[26] Vogelsberger, M., Zavala, J. and Loeb, A. (2012) Monthly Notices of the Royal Astronomical Society, 423, 3740-3754. https://doi.org/10.1111/j.1365-2966.2012.21182.x

[27] Shao, S., Gao, L., Theuns, T. and Frenk, C.S. (2013) Monthly Notices of the Royal Astronomical Society, 430, 2346-2358. https://doi.org/10.1093/mnras/stt053

[28] Vogelsberger, M., Genel, S., Sijacki, D., Torrey, P., Springel, V., et al. (2013) 
Monthly Notices of the Royal Astronomical Society, 436, 3031-3067. https://doi.org/10.1093/mnras/stt1789

[29] Vogelsberger, M., Genel, S., Springel, V., Torrey, P., Sijacki, D., et al. (2014) Nature, 509, 7499-7531. https://doi.org/10.1038/nature13316

[30] Cyr-Racine, F.-Y., Sigurdson, K., Zavala, J., Bringmann, T., Vogelsberger, M., et al. (2016) Physical Review D, 93, Article ID: 123527. https://doi.org/10.1103/PhysRevD.93.123527

[31] Robertson, A., Massey, R. and Eke, V. (2017) Monthly Notices of the Royal Astronomical Society, 465, 569-587. https://doi.org/10.1093/mnras/stw2670

[32] Brinckmann, T., Zavala, J., Rapetti, D., Hansen, S.H. and Vogelsberger, M. (2018) Monthly Notices of the Royal Astronomical Society, 474, 746-759. https://doi.org/10.1093/mnras/stx2782

[33] Vogelsberger, M., Zavala, J., Schutz, K. and Slatyer, T.R. (2019) Monthly Notices of the Royal Astronomical Society, 484, 5437-5453. https://doi.org/10.1093/mnras/stz340

[34] Zavala, J. and Frenk, C.S. (2019) Galaxies, 7, 81-135. https://doi.org/10.3390/galaxies7040081

[35] Iršič, V., Viel, M., Haehnelt, M.G., Bolton, J.S., Cristiani, S., et al. (2017) Physical Review D, 96, Article ID: 023522.

[36] Hsueh, J.-W., Enzi, W., Vegetti, S., Auger, M.W., Fassnacht, C.D., et al. (2019) Monthly Notices of the Royal Astronomical Society, 492, 3047-3059.

[37] Tanabashi, M., Hagiwara, K., Hikasa, K., Nakamura, K., Sumino, Y., et al. (2018) Physical Review D, 98, Article ID: 030001, Section 9.

[38] Holmes, R. (2018) A Quantum Field Theory with Permutational Symmetry. Lambert Academic Press, Riga.

[39] Tanabashi, M., Hagiwara, K., Hikasa, K., Nakamura, K., Sumino, Y., et al. (2018) Physical Review D, 98, Article ID: 030001, Section 14.

[40] Husdal, L. (2016) Galaxies, 4, 78-107. https://doi.org/10.3390/galaxies4040078

[41] Tanabashi, M., Hagiwara, K., Hikasa, K., Nakamura, K., Sumino, Y., et al. (2018) Physical Review D, 98, Article ID: 030001, Section 2.

[42] Merritt, D., Graham, A.W., Moore, B., Diemand, J. and Terzic, B. (2006) The Astronomical Journal, 132, 2685-2700. https://doi.org/10.1086/508988

[43] Ringwald, A. and Wong, Y.Y.Y. (2004) Journal of Cosmology and Astroparticle Physics, 12, Paper 005. https://doi.org/10.1088/1475-7516/2004/12/005

[44] Lane, J.H. (1870) American Journal of Science, 2, 57-74. https://doi.org/10.2475/ajs.s2-50.148.57

[45] Hernquist, L. (1990) The Astrophysical Journal, 356, 359-364. https://doi.org/10.1086/168845

[46] Navarro, J.F., Frenk, C.S. and White, S.D.M. (1996) The Astrophysical Journal, 462, 563-575. https://doi.org/10.1086/177173

[47] Graham, A.W. and Driver, S.P. (2005) Publications of the Astronomical Society of Australia, 22, 118-127. https://doi.org/10.1071/AS05001

[48] Sérsic, J.L. (1963) Boletín de la Asociación Argentina de Astronomía, 6, 41-43.

[49] Einasto, J. (1965) Kinematics and Dynamics of Stellar Systems. Trudy Institute Astrofizica Alma-Ata, Vol. 5, 87-xx.

[50] Graham, A.W. and Spitler, L. (2009) Monthly Notices of the Royal Astronomical 
Society, 397, 2148-2162. https://doi.org/10.1111/j.1365-2966.2009.15118.x

[51] Weinzirl, T., Jogee, S., Khochfar, S., Burkert, A. and Kormendy, J. (2009) The Astrophysical Journal, 696, 411-447. https://doi.org/10.1088/0004-637X/696/1/411

[52] Holmes, R.B. (2020) Journal of Modern Physics, 11, 648-667. https://doi.org/10.4236/jmp.2020.115042

[53] Chandrasekhar, S. (1931) The London, Edinburgh, and Dublin Philosophical Magazine and Journal of Science, 48, 592-596. https://doi.org/10.1080/14786443109461710

[54] Koester, D. and Chanmugam, G. (1990) Reports on Progress in Physics, 53, 837-915. https://doi.org/10.1088/0034-4885/53/7/001

[55] Ruffert, M. and Janka, H.-T. (2010) Astronomy and Astrophysics, 514, A66. https://doi.org/10.1051/0004-6361/200912738

[56] Cho, H.S. and Lee, C.H. (2010) Publications of the Astronomical Society of Japan, 62, 315-321. https://doi.org/10.1093/pasj/62.2.315

[57] Ozel, F. and Freire, P. (2016) Annual Reviews of Astronomy and Astrophysics, 54, 401-440. https://doi.org/10.1146/annurev-astro-081915-023322

[58] von Weizsäcker, C.F. (1935) Zeitschrift für Physik, 96, 431-458. https://doi.org/10.1007/BF01337700

[59] Read, J. (2014) Journal of Physics G. Nuclear Particle Physics, 41, Article ID: 063101. https://doi.org/10.1088/0954-3899/41/6/063101

[60] Kafle, P.R., Sharma, S., Lewis, G.F. and Bland-Hawthorn, J. (2014) The Astrophysical Journal, 794, 59. https://doi.org/10.1088/0004-637X/794/1/59

[61] Prugniel, P. and Simien, F. (1997) Astronomy and Astrophysics, 321, 111-122.

[62] McMillan, P.J. (2011) Monthly Notices of the Royal Astronomical Society, 414, 2446-2457. https://doi.org/10.1111/j.1365-2966.2011.18564.x

[63] de Blok, W.J.G. (2010) Advances in Astronomy, 2010, Article ID: 789293. https://doi.org/10.1155/2010/789293

[64] Oh, S.-H., Hunter, D.A., Brinks, E., Elmegreen, B.G., Schruba, A., et al. (2015) The Astronomical Journal, 149, 180-276. https://doi.org/10.1088/0004-6256/149/6/180

[65] Graham, A., Merritt, D., Moore, B., Diemand, J. and Terzic, B. (2006) The Astronomical Journal, 132, 2701-2710. https://doi.org/10.1086/508990

[66] Vitvitska, M., Klypin, A.A., Kravtsov, A.V., Wechsler, R.H., Primack, J., et al. (2002) The Astrophysical Journal, 581, 799-809. https://doi.org/10.1086/344361

[67] Maccio, A.V., Dutton, A.A., van der Bosch, F.C., Moore, B., Potter, D., et al. (2007) Monthly Notices of the Royal Astronomical Society, 378, 55-71. https://doi.org/10.1111/j.1365-2966.2007.11720.x

[68] Book, L.G., Brooks, A., Peter, A.H.G., Benson, A.J. and Governato, F. (2011) Monthly Notices of the Royal Astronomical Society, 411, 1963-1979. https://doi.org/10.1111/j.1365-2966.2010.17824.x

[69] Bett, P., Eke, V., Frenk, C.S., Jenkins, A. and Okamoto, T. (2010) Monthly Notices of the Royal Astronomical Society, 404, 1137-1156.

[70] Liao, S., Chen, J. and Chu, M.-C. (2017) The Astrophysical Journal, 844, 86-96. https://doi.org/10.3847/1538-4357/aa79fb

[71] Vogelsberger, M., Helmi, A., Springel, V., White, S.D.M., Wang, J., et al. (2009) Monthly Notices of the Royal Astronomical Society, 395, 797-811. https://doi.org/10.1111/j.1365-2966.2009.14630.x 
[72] Tremaine, S. and Gunn, J.E. (1979) Physical Review Letters, 42, 407-410. https://doi.org/10.1103/PhysRevLett.42.407

[73] Grupen, C. (2005) Astroparticle Physics. Springer, Berlin.

[74] Kelso, C., Savage, C., Sandick, P., Freese, K. and Gondolo, P. (2018) European Journal of Physics C, 78, Paper 223. https://doi.org/10.1140/epjc/s10052-018-5685-4

[75] Perkins, D.H. (2000) Introduction to High Energy Physics. 4th Edition, Cambridge University Press, Cambridge.

[76] Patrignani, C., Agashe, K., Aielli, G., Amsler, C., Antonelli, M., et al. (2016) Chinese Physics C, 40, Article ID: 100001, Section 14. https://doi.org/10.1088/1674-1137/40/10/100001

[77] Sterman, G. and Weinberg, S. (1977) Physical Review Letters, 39, 1436-1439. https://doi.org/10.1103/PhysRevLett.39.1436

[78] White, F.M. (1986) Fluid Mechanics. McGraw-Hill, New York.

[79] Johnston, K.V., Majewski, S.R., Siegel, M.H., Reid, I.N. and Kunkel, W.E. (1999) Astronomical Journal, 118, 1719-1726. https://doi.org/10.1086/301037

[80] Law, D.R. and Majewski, S.R. (2010) The Astrophysical Journal, 714, 229-254. https://doi.org/10.1088/0004-637X/714/1/229

[81] Randall, S.W., Markevitch, M., Clowe, D., Gonzalez, A.H. and Bradac, M. (2008) The Astrophysical Journal, 679, 1173-1180. https://doi.org/10.1086/587859

[82] Agrawal, P., Cyr-Racine, F.-Y., Randall, L. and Scholtz, J. (2017) Journal of Cosmology and Astroparticle Physics, 17, Paper 022. https://doi.org/10.1088/1475-7516/2017/05/022 


\section{Appendix A: Estimate of Binding Energy of Hadronic Neutrinos and $\mathrm{SU}(3)_{\text {ve }}$ Interaction Strength}

This Appendix estimates the binding energy of baryonic and mesonic neutrinos as well as the $\mathrm{SU}(3)_{v e}$ interaction strength for relativistic neutrinos. Because the $\mathrm{SU}$ (3) binding energy is a large fraction of the mass-energy of bound quarks, one might expect that this would be the case for SU(3)-bound neutrinos as well (should they exist). This fact is utilized for estimation of the mass-energy of bound neutrino states.

The binding energy of baryonic neutrinos is estimated first. From equation (10.27a) of [38], the binding energy $E_{b}$ of a baryonic neutrino can be approximated by

$$
E_{b}=\beta_{v \tau}^{2}(\hbar c / 4)\left\{4 \pi \alpha_{3}\left(m_{v e}^{2}+m_{v \mu}^{2}+m_{v \tau}^{2}\right)^{1 / 2} c^{2} /(\hbar c)\right\}^{2}|\Delta x|,
$$

where $\beta_{v \tau}^{2}$ is the probability of an upper-mass tau neutrino state (assuming the normal hierarchy), and $\alpha_{3}$ is the dimensionless coupling parameter for the strong force, $g_{s}^{2} /(4 \pi \hbar c)$. The neutrino flavor masses are $m_{v e}, m_{v \mu \nu}$ and $m_{v \tau}|\Delta x|$ is the characteristic size of an SU(3)-bound neutrino. The value of $\alpha_{3}$ is chosen to equal 1 in this calculation because for bound SU(3) states the coupling parameter is close to 1 for bound quark-quark interactions, and that should apply here as well. The probability of an upper-mass neutrino state from the same reference for a marginally relativistic bound state is given by

$$
\beta_{v \tau}^{2}=m_{v e} /\left(m_{v e}+m_{v \tau}\right) .
$$

This probability is approximately 0.1 for $m_{v e} \sim 0.005 \mathrm{eV} / \mathrm{c}^{2}$ and $m_{v \tau} \sim 0.05$ $\mathrm{eV} / \mathrm{c}^{2}$, assuming the normal hierarchy for neutrino masses, the known mass-squared differences, and the least possible mass for the tau neutrino. Under the same assumptions, the muon neutrino mass is about $0.01 \mathrm{eV} / \mathrm{c}^{2}$. The last input to Equation (A1) is the characteristic size of SU(3)-bound neutrinos. For this, use an estimate based on the Heisenberg uncertainty principle:

$$
|\Delta x| \geq \hbar c /(p c) \approx \hbar c /\left(m_{v \tau} c^{2}\right) .
$$

Using the nominal value of $m_{v \tau}$ given above, one obtains $|\Delta x| \sim 3.3$ microns. One might also use $m_{v e}$ or $m_{v \mu}$ in Equation (A3), but the basis of Equation (A1) suggests that $m_{v \tau}$ should be used. Substituting the above into Equation (A1), one obtains an estimate of the binding energy of baryonic neutrinos.

$$
E_{b} \geq 4 \pi^{2} m_{v e} c^{2}=0.2 \mathrm{eV} .
$$

One can see that with these approximations and assumptions, the binding energy is roughly independent of the upper neutrino mass value. In Equation (A3), one might also use $\left(m_{v \tau} m_{v e}\right)^{1 / 2} c^{2}$ for the denominator based on Ch. 10 of [38]. With this assumption, one obtains

$$
E_{b} \geq 4 \pi^{2} m_{v e} c^{2}\left(m_{v \tau} / m_{v e}\right)^{1 / 2}=4 \pi^{2}\left(m_{v \tau} m_{v e}\right)^{1 / 2} c^{2}=0.62 \mathrm{eV} .
$$

To this range of binding energies, one must add the masses of the constituent 
neutrinos, which might range from $3 m_{v e}$ to $3 m_{v r}$ This then leads to a range of baryonic neutrino masses from about $0.22 \mathrm{eV} / \mathrm{c}^{2}$ to about $0.8 \mathrm{eV} / \mathrm{c}^{2}$. Assuming the baryonic neutrinos comprise the lower-mass neutrino states as in quarks, a tighter range would be 0.22 to $0.64 \mathrm{eV} / \mathrm{c}^{2}$. On the other hand, a baryonic neutrino mass as high as $0.8 \mathrm{eV} / \mathrm{c}^{2}$ should not immediately be ruled out. Equation (10.27b) of [38] gives a similar equation for mesonic neutrino states, and the corresponding range of masses is 0.08 to $0.35 \mathrm{eV} / \mathrm{c}^{2}$.

The above mass-scaling analysis can also be applied to relativistic particles using Equation (10.13b) rather than (10.13a) of [38]. In this limit, $\beta_{v \tau}^{2} \sim 0.5$, independent of the interacting masses. Referring to Equation (A1) and removing the $|\Delta x|$ to obtain the interaction force coupling parameter, one finds that the interaction scales as $m_{v \tau}^{2}$ in this case. One then has a scaling factor of $\left(m_{v \tau} / m_{b}\right)^{2}$ to $\left(m_{v \tau} / m_{t}\right)^{2}$ of the quark interaction strength to the neutrino family interaction strength, scaling from the down family or the up family, respectively. Here $m_{b}$ is the bottom quark mass and $m_{t}$ is the top quark mass. Note that the scaling factor between the down-quark family and up-quark family should be of order 1 because all hadrons bound by a strong quark interaction have sufficient energy for the presence of both $u-\bar{u}$ and $d-\bar{d}$ sea quarks. There is also the running of the coupling parameter that should be included; the standard calculation indicates that the correction is negligible when applied to neutrinos. 